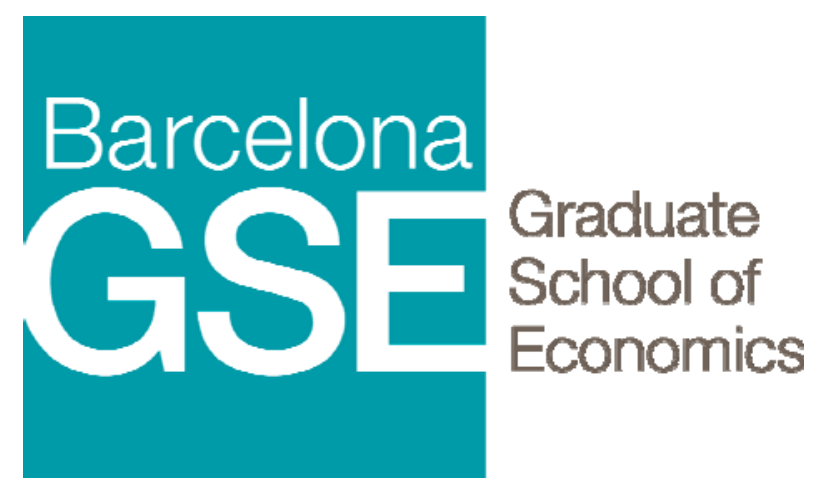

\title{
Coalitional Bargaining with Consistent Counterfactuals
}

Roberto Burguet Ramon Caminal

This version: June 2018 (September 2016)

Barcelona GSE Working Paper Series

Working Paper no 923 


\title{
Coalitional Bargaining with Consistent Counterfactuals*
}

\author{
Roberto Burguet ${ }^{\dagger} \quad$ Ramon Caminal ${ }^{\ddagger}$
}

June 2018

\begin{abstract}
We propose a new solution concept for TU cooperative games in characteristic function form, the $S C O O P$, that builds on the symmetric Nash Bargaining Solution (NBS), adding to it a consistency requirement for negotiations inside every coalition. The $S C O O P$ specifies the probability of success and the payoffs to each coalition. Players share the surplus of a coalition according to the NBS, with disagreement payoffs that are computed as the expectation of payoffs in other coalitions, using some common probability distribution, which in turn is derived from the prior distribution. The predicted outcome can be probabilistic or deterministic, but only an efficient coalition can succeed with probability one. We discuss necessary and sufficient conditions for an efficient solution. In either case, the SCOOP always exists, is generically unique for superadditive games, and easy to compute. Moreover, in the spirit of the Nash program, we propose a reasonable non-cooperative protocol whose stationary equilibrium identifies the SCOOP as the limit equilibrium outcome.
\end{abstract}

KEYWORDS: cooperative games, coalitional bargaining, endogenous disagreement payoffs, consistent beliefs.

JEL classification numbers: C71,C78.

*We are grateful to Francis Bloch, Jean-Jacques Herings, Matthew Ellman, Andreu Mas-Colell and, especially, József Sákovics and Eduard Talamàs, for their useful comments. We acknowledge the support of the Spanish Ministry of Finance, Industry, and Competitiveness (ECO2017-88129-P, and Severo Ochoa Programme for Centres of Excellence in R\&D, SEV-2015-0563) and Generalitat de Catalunya (2017-SGR1136).

†University of Central Florida, Institute of Economic Analysis (CSIC), and Barcelona GSE, burguet@ucf.edu

${ }^{\ddagger}$ Institute of Economic Analysis, CSIC, and Barcelona GSE, ramon.caminal@iae.csic.es 


\section{Introduction}

The Nash Bargaining Solution (NBS) has long been accepted as the standard solution concept for negotiations between two, or more, players about how to share the proceeds of an agreement. Less consensus exists as to the right theory for the problem of coalition formation. There, different possible agreements involve different sets of players, each characterized by the worth that the players in the set may obtain and share, should they reach that agreement. For this problem, we propose a theory that builds on the symmetric NBS, ${ }^{1}$ adding to it a consistency requirement for negotiations inside every coalition. More specifically, we study the problem of forming one of the possible coalitions of a TU game in characteristic function form. We propose a new solution concept, the SOlution with Consistent Outside Options (SCOOP), that specifies the probability of success of, as well as the payoffs within, each coalition. It requires that the surplus in every coalition is shared according to the NBS, with disagreement points consistently computed. In particular, each player's disagreement payoff in one coalition is that player's expected payoff in alternative coalitions. The expectation is taken using some probability distribution over those counterfactuals, ${ }^{2}$ a system of beliefs that is shared by all players and is consistent across coalitions. That is, the system of beliefs coincides with the system of conditional distributions obtained from a joint distribution, a common prior, over all coalitions. The theory identifies that system of beliefs and that common prior. Finally, we also require that the probabilities are consistent with the payoffs: a coalition may be expected to succeed with positive probability only if all players involved have no better option.

Our first major result is that the SCOOP exists for any game. That is, for any transferable-utility game of coalition formation there exists a, perhaps probabilistic, prediction of success and surplus sharing consistent with NBS, with the disagreement payoffs determined by consistent counterfactuals.

We then turn to investigate uniqueness. Restricting attention to superadditive games, we show that the SCOOP is generically unique. ${ }^{3}$ Thus, for superadditive games the

\footnotetext{
${ }^{1}$ Like in most applications of the NBS, players are assumed to equally share the surplus net of the disagreement payoffs.

${ }^{2}$ We refer to these counterfactuals as outside options, although they are external only when considered from the point of view of negotiations to reach a particular agreement or form a particular coalition.

${ }^{3}$ Uniqueness can also be ascertained for other classes of games like indivisible games (any pair of coalitions with positive worth share at least one player), like the three-player, three-cake problem, or symmetric games (the worth of the coalition only depends on its size).
} 
SCOOP provides sharp predictions which, moreover, are relatively straightforward to compute. We also argue that, maintaining the assumption that only one coalition forms, any sensible solution should content itself with less sharp predictions for more general classes of games. As a simple illustration, if the only coalitions with positive surplus are two disjoint coalitions, there is nothing in the characteristic function form on which to base any prediction sharper than this: one of these two coalitions will form.

We also study the nature of the predictions that follow from our theory. It should not come as a surprise that when the SCOOP is deterministic, i.e., predicts the formation of a certain coalition with probability one, such a coalition must be the grand coalition in strictly superadditive games; or, more generally, one with maximum worth (efficient). Having a non-empty core is a necessary, but not sufficient, condition for a game to have a deterministic SCOOP. Indeed, a deterministic SCOOP requires that the payoffs to the players in the grand coalition are preferable to what they might obtain elsewhere. This is also what an imputation must satisfy to be in the core, and so the payoffs in a deterministic SCOOP must be an allocation in the core. An immediate implication is that, if the core is empty, the SCOOP is always probabilistic.

However, a deterministic SCOOP requires more than a non empty core. In particular, it requires that the agreement in the efficient coalition be supported by consistent counterfactuals, a stronger requirement, and one which all core allocations may fail to satisfy. Thus, even if the core of the game is non-empty, the SCOOP may be probabilistic. We characterize the set of games for which the SCOOP is deterministic and the set for which it is probabilistic.

In line with the Nash program, we also offer a non-cooperative protocol that implements the SCOOP. First, we discuss why (versions of) the two standard protocols, the random-proponent protocol and the rejector-proposes protocol, may fail to produce satisfactory predictions. ${ }^{4}$ Indeed, besides possibly generating multiple equilibria, these protocols include initial exogenous (i.e., arbitrary) random devices that end up having a large impact on the predicted outcomes. In particular, the equilibrium probabilities of success may be insensitive to changes in the primitives, and exclusively determined by these exogenous random devices. Our approach is to construct a protocol with elements of these classes of protocol, but less bonded to the random device. More specifically, our protocol departs from the standard ones by, first, separating the determination of the

\footnotetext{
${ }^{4}$ See Ray (2007) for an excellent discussion of these protocols and other related issues.
} 
winning coalition from the sharing of the value of this coalition; and, second, by endogenizing the probabilities with which the candidate winning coalition is determined. In our protocol, stationary equilibria (exist and) necessarily identify the SCOOP as the limit equilibrium outcome.

One early predecessor of our paper is Bennett (1997), who also endogenizes disagreement payoffs in a particular negotiation as functions of the payoffs that players obtain in their alternative negotiations. For the three-player/three-cake problem (Binmore, 1985), Bennett's approach is to consider as disagreement payoffs in each bilateral negotiation the payoffs that each player obtains in their negotiations with the third player. In our language, this is equivalent to assuming that players assign probability one to several, mutually exclusive, possible counterfactuals. The SCOOP follows Bennett's lead but adds the requirement that the counterfactuals used to compute disagreement points be consistent.

The idea of modeling the division of surplus as based on endogenous disagreement points is also at the heart of various definitions of internal consistency (the reduced-game property) that have been invoked to characterize the nucleolus and the Shapley value as appropriate generalizations of the NBS (Sobolev, 1975; Peleg, 1986; Hart and MasColell, 1989; Serrano and Shimomura, 1998). Here too, the reduced game is defined while overlooking the possible incompatibility of alternative agreements. For instance, in a three-player game, following Hart and Mas-Colell (1989), the Shapley value is consistent in the following sense: the payoffs to every pair of players is also the NBS of their bilateral negotiation, with disagreement points defined as the payoffs that each player obtains in case this negotiation fails and reaches an agreement with the third player. Clearly, these disagreement payoffs are not based on consistent counterfactuals, since only one of the players can possibly reach an agreement with the third.

The more recent paper by Compte and Jehiel (2010) is also related to the present paper. In the context of games in characteristic function form in which the grand coalition is the one that generates the largest surplus, they define a cooperative solution concept, the Coalitional Nash Bargaining Solution (CNBS), as the allocation in the core that maximizes the Nash product. (Thus, their concept is only defined for games with a non-empty core.) The SCOOP coincides with the CNBS when the former is deterministic. However, as we have mentioned above, even when the core is not empty, and so the CNBS is defined, the SCOOP may be probabilistic, and so predict a quite different outcome. Once more, the 
requirement that payoffs are based on the NBS and consistent counterfactuals explain the departure. Interestingly, there is a property that Compte and Jehiel discuss that we use to characterize the class of games for which the SCOOP is deterministic. ${ }^{5}$

The rest of the paper is organized as follows. In the next section we present our solution concept. Sections 3 and 4 examine the existence and uniqueness of a SCOOP. The non-cooperative implementation is discussed in Section 5. Section 6 illustrates the main characteristics of the SCOOP with some examples. Section 7 discusses the relation between the SCOOP, the core, and the CNBS. The paper closes with precise guidelines on the computation of the SCOOP in complex games (Section 8), and some concluding remarks (Section 9).

\section{A new solution concept for cooperative games in characteristic function form}

We consider $n>2$ agents who negotiate to form a coalition. The set, and the coalition, of all agents will be denoted by $N$, and the set of coalitions by $S$. The coalition $s \in S$ has worth $v_{s} \geq 0$. Thus, the pair $\left(N,\left\{v_{s}\right\}_{s \in S}\right)$ defines a coalitional game: a cooperative game in characteristic function form. In this paper we study the problem of forming a unique coalition and distributing its worth among its members.

Thus, a solution identifies an outcome. A deterministic outcome is a coalition $s \in S$, and payoffs, $u_{i}^{s}$, for all $i \in s$. We will also allow for non-deterministic outcomes. Thus, in general, an outcome is a probability distribution $\mathbf{p}$ over the set of deterministic outcomes.

The elements of our theory are simple: agents will consider all possible negotiations (one in each coalition $s \in S$ ) simultaneously. Payoffs within each coalition must correspond to the symmetric (equal sharing of the net surplus) Nash Bargaining Solution inside the coalition, and the disagreement points for each of these negotiations must correspond to the (expected) payoffs in alternative coalitions. Finally, the expectation is computed using Bayes' rule and a common probability distribution over coalitions. This probability distribution must be consistent with the agents' payoffs in all coalitions.

We normalize to 0 the payoff of a player that does not belong to the coalition that is formed. (Note that this does not exclude the possibility that one-player coalitions have

\footnotetext{
${ }^{5}$ This is not coincidence: that property, referred to as Property P1, guarantees that the CNBS is the subgame equilibrium (limit) outcome of a random proponent protocol. Subgame perfection in fact incorporates consistency of beliefs, very much in line with the consistency requirement for the SCOOP.
} 
positive worth.) Let $\mathbf{p} \equiv\left\{p_{s}\right\}_{s \in S}$ denote a probability distribution on $S$. Also, for each $s$, let $\left\{\lambda_{r}^{s}\right\}_{r \neq s}$ denote a probability distribution on the set of coalitions minus $s$. Finally, for each $s \in S$, denote by $\left\{t_{i}^{s}\right\}_{i \in s}$ a vector of disagreement points for the $n_{s}$ players in coalition $s$.

Definition A Solution with Consistent Outside OPtions $(S C O O P)$ for the game $\left(N,\left\{v_{s}\right\}_{s \in S}\right)$ is a quadruple $\sigma=\left\{\left\{u_{i}^{s}\right\}_{i \in s},\left\{t_{i}^{s}\right\}_{i \in s}, p_{s},\left\{\lambda_{r}^{s}\right\}_{r \neq s}\right\}_{s \in S}$ that satisfies:

(i) $u_{i}^{s}=\left\{\begin{array}{lr}t_{i}^{s}+\frac{1}{n_{s}}\left(v_{s}-\sum_{j \in s} t_{j}^{s}\right), \text { if } v_{s} \geq \sum_{j \in s} t_{j}^{s} \\ 0, & \text { otherwise }\end{array}\right.$

(ii) $t_{i}^{s}=\sum_{r \ni i, r \neq s} \lambda_{r}^{s} u_{i}^{r}$. If $p_{s}<1$ then $\lambda_{r}^{s}=\frac{p_{r}}{1-p_{s}}$.

(iii) $p_{s}>0$ only if $u_{i}^{s} \in \max _{r \ni i} u_{i}^{r}$, for all $i \in s$.

The first line in (i) is simply the symmetric Nash Bargaining Solution. The second line corresponds to the "outside-option principle": $u_{i}^{s}$ may play (part of) the role of an outside option for an agent $i \in s$ in negotiations elsewhere, but only if $s$ is indeed a coalition that $i$, and the rest of members of $s$, may consider joining. Otherwise, the payoffs in $s$ must be irrelevant, i.e., add nothing to the agents' disagreement points when they negotiate to form other coalitions $r \neq s$.

Part (ii) in the definition establishes that the disagreement points in $s$ are the expected payoffs in alternative negotiations $r \neq s$. It also requires that the probability distribution used to calculate these expected payoffs in alternative negotiations is consistent. That is, it must be the conditional probability distribution over coalitions other than $s$ obtained from a common prior $\mathbf{p}$ using Bayes' rule, when applicable. That is, the SCOOP can be probabilistic, $p_{s}<1$ for all $s$, in which case $\lambda_{r}^{s}$ is given by Bayes' rule, or deterministic, $p_{s}=1$ for some $s$, in which case $\lambda_{r}^{s}$ can be freely chosen.

Part (iii) imposes the consistency of this common prior with payoff predictions. A coalition can have positive probability only if all its members obtain their maximal payoff in that coalition. That is, any player is expected to reject a coalition if there is an alternative one that she strictly prefers. ${ }^{6}$

Thus, our solution concept can be understood as a generalization of the (symmetric) NBS to coalitional games, in which the beliefs held in each coalition are consistent: (a) all players in each coalition hold the same beliefs about the consequences of the failure

\footnotetext{
${ }^{6}$ Weaker consistency criteria may be defended. Yet, this strong one is not only compatible with existence, but delivers a unique prediction in a large class of games. Weaker criteria would have to include this prediction, and so it may be argued that the SCOOP would still be the most robust prediction.
} 
of the negotiations, (b) these beliefs emanate from a common probability distribution (and hence they are consistent across coalitions), and (c) this probability distribution is compatible with the payoffs. In Section 5, we study a non-cooperative, dynamic bargaining game that implements, as the discount vanishes, the SCOOP. The bargaining game is a sensible version of standard protocols, in particular, random-proponent and rejectorproposes protocols.

\section{Existence}

Our first result guarantees that this concept always results in a prediction. That is, for any game in characteristic function form, it is possible to model simultaneous negotiations à la Nash with consistent counterfactuals and obtain some prediction, a SCOOP. Indeed,

Theorem 1 For any TU game, a SCOOP exists.

The proof is in the Appendix. In this general existence proof, we have used an argument that parallels the by now most popular proof of the existence of a competitive (Walrasian) equilibrium. The similarity comes from the use of (a version of) the Walras law. Indeed, existence of a competitive equilibrium can be proved by referring to (continuity and) the Walras law: the product of any vector of prices and the corresponding excess of demand over endowments is always zero. These properties are used to define a correspondence from the set of (relative) price vectors to itself, a fixed point of which is a competitive equilibrium. For the SCOOP, the analogues of prices are the probabilities. "Excess demand" in each "market" (coalition) has nothing to do with utility maximization, but simply with a particular property of the SCOOP (the "equal loss"), which is a direct consequence of the "behavior" predicted by the - symmetric - Nash bargaining theory. Crucially, this "excess demand" also satisfies an analogue to the Walras law: the expected total excess payoff is always zero. Using (continuity and) this property, we also define a correspondence from the set of probability vectors to itself a fixed point of which is also virtually the SCOOP. This is where the parallel ends. However, just as with competitive equilibrium, it is instructive to view the (Kakutani's) fixed point theorem as a process of convergence. In our case, the "Walrasian auctioneer" would name a vector of probabilities, check where there is "excess demand", i.e., in what coalitions the payoffs induced by equal loss exceed the worth of the coalition, and then try a new probability 
vector that puts less probability on those coalitions. ${ }^{7}$

\section{Uniqueness}

Our second main result refers to the sharpness of the predictions that the SCOOP offers. For that, we will restrict attention to superadditive games.

Definition The game $\left(N,\left\{v_{s}\right\}_{s \in S}\right)$ is superadditive if for all $s, s^{\prime} \in S$ such that $s \cap s^{\prime}=\varnothing$, $v_{s \cup s^{\prime}} \geq v_{s}+v_{s^{\prime}}$.

Moreover, we will consider games where $v_{\{i\}}=0$ for all $i .^{8}$ That is, games where a true "agreement" (i.e., one among at least two players) is needed to create a surplus above what individual players obtain when no coalition (that includes them) forms.

In this set of games, multiplicity only arises for very particular combinations of coalition values; that is, for a subset of games with zero measure in the set of superadditive games.

Theorem 2 Generically, the SCOOP of a superadditive game is unique.

The proof is in the Appendix. We offer here a sketch of the strategy. First, suppose that there is a SCOOP in which the grand coalition - which is generically the only efficient coalition - succeeds with probability 1 . In this case, part (iii) of the definition of a SCOOP implies that the worth of any other coalition should be lower than or equal to the sum of the payoffs that its members obtain in the grand coalition. Also, the second line of part (i) of the definition — which embeds the outside-option principle - implies that the only coalitions that can affect the distribution of payoffs in the grand coalition are the ones in which the above weak inequality holds with equality. These two points, together with the principle that players equally share the net surplus — first line of part (i) —, imply that the payoff vector of a deterministic SCOOP is in fact the imputation in the core that

\footnotetext{
${ }^{7}$ The proof in fact shows not only that a SCOOP exists, but also that one exists that satisfies the - conditional or not- "equal loss" that we discuss later. This has some interest, as our generic uniqueness result in the next section is solely based on this equal loss. That is, the SCOOP identified in this proof is generically unique in the set of all games.

${ }^{8}$ Note that the worth of an individual coalition may be different from the payoff to the individual when she is not a member of the winning coalition. Thus, we are making the, often implicitly made, assumption that the game is of strict coalition formation. That is, a coalition needs to have more than one member to produce any surplus. This assumption is not really necessary for uniqueness, but rather helps simplifying the analysis of the strategic implementation of the SCOOP.
} 
maximizes the Nash product. Since the Nash product is strictly quasiconcave and the set defined by the inequality constraints implied by part (iii) - the core - is convex, the solution is unique.

This is the way the CNBS of the game (Compte and Jehiel, 2010) is defined and so this already implies that, when the SCOOP is deterministic, the SCOOP coincides with the CNBS. However, as we will discuss in more depth in Section 7, even when the CNBS exists - i.e., when the core is not empty - the SCOOP may not be deterministic. That is, there may not be consistent counterfactuals that sustain the CNBS as a SCOOP.

Second, suppose that there is a probabilistic SCOOP that puts some positive probability only on coalitions in some set $S_{+}$, which cannot include the grand coalition. Part (iii) of the definition of the SCOOP implies that all these coalitions containing player $i$ must offer player $i$ the same payoff, say $u_{i}$. We can now define a new game where the grand coalition has a worth equal to the sum of $u_{i}$ for all players. Consider the same constrained optimization problem described in the previous paragraph for this modified game. The payoffs predicted by the SCOOP in the original game must be a solution, and so the unique solution, of this problem! Indeed, the first order conditions of the optimization problem are simply — part of - the conditions that define the SCOOP with only interpreting probabilities as - multiples of - Lagrange multipliers. Thus, we only need to show that there can be no two such modifications of the original game, as the solution to one would induce slack - and so, a contradiction to the first part of the definition of the $\mathrm{SCOOP}$ - in an active constraint of the other.

The above discussion already suggests that those games that posses a deterministic SCOOP typically cannot posses a probabilistic one, and viceversa. More specifically, the intersection of the set of games with a deterministic SCOOP and the set of games with a probabilistic SCOOP is not empty but has an empty interior.

Theorem 2 is remarkable. For superadditive games, the consistency of the counterfactuals that satisfy the particular way in which — symmetric - Nash bargaining theory predicts the sharing of surplus in every negotiation is not only feasible, but - genericallyis compatible with only one - perhaps probabilistic - outcome.

Non-generic multiplicity may arise in two different circumstances. First, in the intersection between the region with a deterministic SCOOP and the region with a probabilistic SCOOP, which as mentioned above has a non-empty interior, there are typically a continuum of SCOOPs. For instance, consider a three-player game with $v_{N}=1$, and $v_{s}=2 / 3$ 
for any coalition $s$ with two players, and with zero-worth individual coalitions. ${ }^{9}$ There exists a deterministic SCOOP with $p_{N}=1$, and $u_{i}=1 / 3$ for all $i$. However, there also exists a continuum of non-deterministic SCOOPs where $p_{N}=p$ for any $p \in[0,1)$, and $p_{s}=(1-p) / 3$ for each two-player coalition $s$. Still, every player that belongs to the winning coalition obtains a payoff of $1 / 3$, but obviously these different SCOOPs are not equivalent. On the contrary, they are Pareto-ranked, as all individual probabilities of success increase with $p$. Thus, in this boundary case, the SCOOP acknowledges a potential coordination problem: the indifference of players between coalitions of different size may preclude a more efficient outcome.

Multiplicity may also arises in games in which more than one coalition of size smaller than $n$ have the same worth as the grand coalition, another example of non-generic games. For instance, consider a four player game where $v_{s}=1$ for $s=\{1,2,3,4\},\{1,2,3\}$, $\{1,2,4\}$, and $v_{s}=0$ for all other coalitions. Any $\left(u_{1}, u_{2}\right)$ such that $u_{1}+u_{2}=1, u_{i} \geq 0$ will be - part of - a SCOOP. Hence, in this type of non-generic game, multiplicity is again payoff relevant. Only players who belong to all coalitions with the maximum worth can obtain positive payoffs, but the mere existence of these alternative efficient coalitions may provide alternative, consistent counterfactuals that sustain different predictions. Thus, in this case, the perfect substitutability of players may leave the vector of payoffs undetermined..$^{10}$

The focus on superadditive games is natural in games without externalities. It is often argued that if $s$ and $s^{\prime}$ are two disjoint coalitions, then they should be able to "...accomplish at least as much by joining forces as by remaining separate" (Owen, 1995, page 213). On the other hand, as we anticipated in the Introduction, in the case of games in which only one coalition can form (that is, the value of all other coalitions drops to zero after one has formed, an extreme type of externality), if the game is not superadditive and disjoint coalitions are relevant, then the multiplicity of SCOOPs should be expected. Indeed, consider a four-player game in which only two coalitions, say $(1,2)$ and $(3,4)$,

\footnotetext{
${ }^{9}$ If $v_{N}>1$ the unique SCOOP is deterministic, $p_{N}=1$, and if $v_{N}<1$ the unique SCOOP is probabilistic, $p_{s}=\frac{1}{3}, p_{N}=0$.

${ }^{10}$ There are other, non generic, examples of irrelevant multiplicity, as all the SCOOPs predict the same payoffs and same probabilities of success for all players. First, there are multiple deterministic SCOOPs in terms of the composition of the set of relevant outside options, $S_{0}(\sigma)$, where these alternative sets represent equivalent disagreement payoffs and hence result in the same payoffs. Similarly, there may be multiple non-deterministic SCOOPs in terms of the identity of the coalitions that succeed with positive probability, but result in the same individual payoffs and the same individual probabilities of belonging to the winning coalition.
} 
have positive worth — provided the other coalition has not been formed. With - onlythis information, it does not seem reasonable to predict which of these two coalitions will win the "race". This is what the SCOOP acknowledges: any probability distribution on these two coalitions may be part of a SCOOP.

Superadditivity is a sufficient but not a necessary condition for (generic) uniqueness. In the concluding section we discuss some classes of games that are not necessarily superadditive and nevertheless exhibit a (generically) unique SCOOP.

\section{Non-cooperative implementation}

Following the Nash program, we should now inquire what type of strategic game may implement our solution concept in the class of superadditive games - where $v_{\{i\}}=0$ for all $i$. We should note that the most popular protocols in the recent bargaining literature (with irreversible agreements) are not well fitted to do the job. Any of these protocols begins with one player, who is chosen using an exogenous random device, proposing a particular coalition and how to share its value. Next, the members of this coalition accept or reject the offer. Different protocols differ with respect to the consequences of a rejection. In the rejector-proposes protocol (Chaterjee et al. 1993), the first player who rejects the offer becomes the next proponent. In the random-proponent protocol (e.g., Compte and Jehiel, 2010), a new proponent is chosen again by the same random device used at the beginning.

These classes of protocols may be too rigid if we want to allow for unrestricted, probabilistic predictions. Indeed, if a player $i$ has some probability of belonging to a coalition that may form and is chosen by Nature as the proponent with probability $\frac{1}{n}$ (and players discount the future, so that everybody approached by the proponent has incentives to accept in equilibrium), it seems difficult to predict that player $i$ has probability less than $\frac{1}{n}$ of belonging to the forming coalition. Such rigidity is quite independent of the primitives of the bargaining setup, and is related only to the model's arbitrary moves by Nature.

Our approach is to study a simple version of these standard protocols with two key modifications. The first is to separate the determination of the -candidate- winning coalition from the sharing of the value of a coalition. The second is to endogenize the probabilities with which the - candidate- winning coalition is determined.

Thus, consider the following bargaining game, which takes place over time: $t=$ $0,1,2, \ldots$ (infinite horizon). All players discount the future using the same discount factor, 
$\delta$. In period $t=0$ :

\section{SELECTING THE COALITION}

(1.1) Each player $i$ simultaneously selects a vote $b_{s}^{i} \geq 0$ for each coalition $s \ni i$ with $n_{s}>1$ and $\sum_{s \ni i} b_{s}^{i} \leq 1$.

(1.2) Nature selects a coalition $s$ according to the probability distribution $\mu$ on $S$, where

$$
\mu_{s}=\frac{\min _{i \in s} b_{s}^{i}}{\sum_{z \in S} \min _{i \in z} b_{z}^{i}},
$$

as long as $\sum_{z \in S} \min _{i \in z} b_{z}^{i}>0$, and otherwise, the game moves to stage (1.1) in the next period.

(1.3) All players involved in coalition $s$ must accept or reject in sequence. a) If all players accept, the game moves to (2). b) If one player rejects, the player selects a coalition $s^{\prime}$ and the game moves to (2.1) in the next period. (It is the player who rejects, not Nature, who proposes the coalition and does not need approval from the rest.)

\section{SHARING THE VALUE OF THE COALITION}

(2.1) Nature selects one of the members of coalition $s$ (or $s^{\prime}$ ), say $j$, as the proponent using a uniform probability distribution. Player $j$ makes a proposal to divide $v_{s}$.

(2.2) The players in $s$ accept or reject in sequence. a) If all accept, then the proposal is implemented and the game ends. Otherwise, the game moves to the next period and starts fresh at (1.1).

At any $t$ reached with no agreement, if in time $t-1$ a player rejected at stage 2 or if $\sum_{z \in S} \min _{i \in z} b_{z}^{i}=0$, then players continue bargaining following the protocol as in $t=0$. If instead an offer was rejected in stage (1.2) of period $t-1$, then the first player who rejected the offer is the current proponent of a coalition in stage (1.2). In line with our restriction to games where a "true" coalition (i.e., between at least two players) is needed to create surplus, in (1.1) players can only vote for such coalitions.

We study subgame perfect equilibria of this game in stationary strategies. We define a strategy for player $i$ to be stationary if the choices in period $t$ are independent of the history of the play up to that period. Moreover, in the same spirit, we require that the answers in stage (1.3) and in stage (2) depend on the selected $s$, but not on anything else, in particular the ballots $b$. (Alternatively, we may suppose that these ballots are not observed by other players.)

Theorem 3 As $\delta \rightarrow 1$, any limit point of the sequence of outcomes (coalition that forms and division of the surplus) of any selection of subgame perfect equilibria in stationary 
strategies, with positive probability of agreement, is the outcome of a SCOOP. Moreover, such sequence of equilibria exists. Thus, asymptotically, the protocol implements the SCOOP.

A formal proof of this result is in the Appendix. Here, we offer a heuristic proof of the "necessity part" for the case, perhaps less intuitive, where the limiting equilibrium puts a positive probability on more than one coalition.

Let $\delta$ be given, and denote the equilibrium payoff of player $j$ by $U_{j}$. Whoever is the proponent in stage (2.1) when coalition $s$ has been accepted, say player $i$, will obtain a premium of $v_{s}-\sum_{j \in s} \delta U_{j}$, above her continuation payoff, $\delta U_{i}$ (if that premium is positive). This premium is the same for all players in $s$, a feature which is shared by all other standard protocols based on proposals. In our protocol, and for sufficiently high $\delta$, any player that belongs to a coalition at which this premium is highest will reject in (1.3) any coalition with lower premium and propose one such premium-maximizing coalition in the next period. (The latter will surely be accepted by all members.) This, together with superadditivity, implies that any non premium-maximizing coalition will be blocked by some player. Put in other words, all coalitions that are accepted with positive probability must be characterized by the same (in the limit) premium. (Obviously, this also implies that coalitions that do not form are characterized by weakly smaller premia, and that payoffs for each player are the same in all of her coalitions that succeed with positive probability.) As we discuss in the Appendix and in the examples in the next section, this implies "equal loss": all players -that belong to a coalition that forms with positive probability - expect to lose the same as a consequence of the probability of not being in the realized winning coalition. This, together with equal payoff for each player in all her coalitions that form with positive probability and no higher payoffs anywhere else, are the defining characteristics of a SCOOP.

\section{Examples}

We now consider a few numerical examples that illustrate the main characteristics of a SCOOP. They also suggest how to compute it. We start with the simplest example: a three-player game.

Example 1. Let $n=3, v_{N}=10, v_{12}=8, v_{13}=v_{23}=2$, where in this and the rest of examples a subscript or upperscript $i j(k)$ refers to the coalition of players $i$ and $j$ (and 
$k$ ). Individual coalitions have zero value: $v_{i}=0, i=1,2,3$. The SCOOP predicts that the grand coalition forms with probability one: $p_{N}=1$. Coalitions $(1,3)$ and $(2,3)$ prove irrelevant and hence they can be ignored as far as the outcome is concerned. Players 1 and 2 are in a symmetric position and hence they must obtain the same: $u_{1}^{N}=u_{2}^{N}$. If each of these players were to obtain less than $4, u_{i}^{N}<4$, then, provided the grand coalition is formed for sure, there would exist a positive net surplus in coalition $(1,2), t_{1}^{12}+t_{2}^{12}$ (= $\left.u_{1}^{N}+u_{2}^{N}\right)<v_{12}$, and hence these two players would prefer to form their own coalition $\left(u_{i}^{12}>u_{i}^{N}, i=1,2\right)$, which would render $p_{N}>0$ impossible (part (iii) of the definition). Instead, if $u_{i}^{N}>4$ then the "threat" of leaving the grand coalition would cease to be credible, since at most each of these players could get 4 in the alternative coalition. That is, $t_{1}^{12}+t_{2}^{12}$ would be higher than $v_{12}$ and hence $u_{1}^{12}=u_{2}^{12}=0$. As a result, $t_{1}^{N}=t_{2}^{N}=0$ and hence their payoffs in the grand coalition would be at most $\frac{10}{3}<4$. Therefore, the SCOOP satisfies the outside option principle. Thus, the grand coalition will form for sure, players 1 and 2 will obtain 4 each, and player 3 will obtain 2 .

For completeness, the beliefs that sustain this outcome as the NBS of the grand coalition include $\lambda_{12}^{N}=\frac{1}{2}$. We can let $\lambda_{i 3}^{N}, i=1,2$, and $\lambda_{j}^{N}, j=1,2,3$ be any non-negative numbers such that they add up to $\frac{1}{2}$. Also, no agreement in coalitions $(1,3)$ and $(2,3)$ is feasible, since $t_{i}^{i 3}+t_{3}^{i 3}=6>v_{i 3} i=1,2$, and hence $u_{i}^{i 3}=u_{3}^{i 3}=0$. Finally, coalition $(1,2)$ is the only influential bilateral coalition, which implies that its net surplus is zero: $t_{1}^{12}+t_{2}^{12}=v_{12}$, and hence $u_{i}^{12}=t_{i}^{12}=4$.

This example illustrates the main characteristics of a deterministic SCOOP, which hold more generally, as proved in Lemma 7 in the Appendix (for the class of superadditive games). First, only an efficient coalition can succeed with probability one. Hence, a deterministic SCOOP is tantamount to an efficient outcome. Second, a deterministic SCOOP is also characterized by the set of relevant outside options. In the example, this set is a singleton (coalition $(1,2)$ ). Even though these coalitions have zero probability of success, they influence the negotiations in the winning coalition. Third, in all other coalitions that represent non-credible outside options, all players would obtain a payoff of zero, since the net surplus is negative. Fourth, the beliefs that support the payoffs in the winning coalition must satisfy the "conditional equal loss" principle: the (counterfactual) expected losses associated with the failure of negotiations in the winning coalition are the same for all its members; that is, $u_{1}^{N}\left(1-\lambda_{N}^{12}\right)=u_{2}^{N}\left(1-\lambda_{N}^{12}\right)=u_{3}^{N}$.

Example 2. Consider the previous example, but with a higher worth of coalitions 
$(1,3)$ and $(2,3): v_{13}=v_{23}=7$. If we were to predict the success of the grand coalition with probability one, then for any distribution of payoffs at least one pair of players would rather leave the grand coalition and form their bilateral coalition. In this game, the SCOOP predicts a probabilistic outcome. In particular, $p_{12}=\frac{4}{10}, p_{13}=p_{23}=\frac{3}{10}$. Of course, players obtain their payoffs only in case they belong to the winning coalition, but if they do, their payoffs are $u_{1}=u_{2}=4, u_{3}=3$, independently of the specific winning coalition. In other words, players 1 and 2 obtain 4 with probability $\frac{7}{10}$ (and zero with probability $\frac{3}{10}$ ) and player 3 obtains 3 with probability $\frac{6}{10}$ (and zero with the complementary probability). Notice that in the grand coalition there is a positive net surplus, but all players obtain a payoff lower than in their alternative coalitions. In particular, $t_{1}^{N}=t_{2}^{N}=\frac{28}{10}, t_{3}^{N}=\frac{18}{10}$. As a result, $\frac{1}{3}\left(v_{N}-\sum_{i} t_{i}^{N}\right)=\frac{13}{15}$, and $u_{1}^{N}=u_{2}^{N}=\frac{11}{3}<4, u_{3}^{N}=\frac{8}{3}<3$.

This example illustrates the main characteristics of a probabilistic SCOOP, which holds more generally, as proved in Lemma 8 in the Appendix. First, any player that belongs to more than one coalition that succeeds with positive probability obtains the same payoffs in all of them. Second, players' payoffs in these coalitions exhaust the full value of the coalition. Third, the probability distribution satisfies the "equal loss" property: the expected loss of each player that belongs to at least one of these coalitions, with respect to the ideal scenario where all players obtain their payoffs with probability one, is the same for all players. Fourth, coalitions with zero probability of success have a worth inferior to the sum of the players' payoffs. ${ }^{11}$

The game in Example 1 has a non-empty core and the payoffs predicted by the (deterministic) SCOOP are a selection of that set. In contrast, the game in Example 2 has an empty core and the SCOOP is probabilistic (and hence, inefficient). For three-player games, this is always so: the SCOOP is deterministic if and only if the game has a nonempty core. For games with more players, the non-emptiness of the core is necessary, but not sufficient, for the SCOOP to be deterministic. Indeed, consider the following example.

Example 3. $n=4, v_{N}=31, v_{123}=30, v_{14}=v_{24}=14$, and the value of all other coalitions is zero. Note that the core is not empty. In particular, $x_{1}=x_{2}=13, x_{3}=4$, $x_{4}=1$ belongs to the core. In fact, if the SCOOP of this game was deterministic then it would be characterized by $p_{N}=1$ and $u_{i}^{N}=x_{i}$. The intuition for such a payoff distribution is the same as in Example 1. All other alternative coalitions are relevant outside options,

\footnotetext{
${ }^{11}$ If a player does not belong to any of the coalitions with a positive probability of success, then we impute a payoff equal to the "equal loss".
} 
and hence payoffs must satisfy $\sum_{s \ni i} u_{i}=v_{s}$ for all these coalitions. However, to sustain these payoffs as the NBS of the negotiations in the grand coalition, players 1 and 2 should have a disagreement payoff of $\frac{429}{35}$, and players 3 and 4 a disagreement payoff of $\frac{104}{35}$ and $\frac{9}{35}$, respectively. That would be necessary for the "conditional equal loss" to be satisfied: $u_{i}^{N}\left(1-\sum_{s \ni i} \lambda_{N}^{s}\right)$ should be the same for all players, which implies that $\lambda_{N}^{123}=\frac{57}{70}, \lambda_{N}^{14}=\lambda_{N}^{24}=\frac{9}{70}$. Notice that with these values $\sum_{s} \lambda_{N}^{s}>1$. That is, there are no consistent beliefs that can sustain that payoff vector. Put in other words, there are no mutually compatible outside options that sustain the core outcome. In particular, in the grand coalition players 1,2 , and 3 can jointly claim a total payoff of 30 , which means that player 4 can at most claim 1 . Given that, both players 1 and 2 could claim 13 each $\left(v_{i 3}-1\right.$, more than their equal share of the worth of coalition $\left.(1,2,3)\right)$. However, there are no beliefs that can sustain all these claims simultaneously.

In contrast, the SCOOP of this game is probabilistic. In particular, $p_{123}=\frac{3}{4}, p_{14}=$ $p_{24}=\frac{1}{8}$, and the payoffs conditional on success are $u_{1}=u_{2}=12, u_{3}=6, u_{4}=2$.

\section{More on the core: The CNBS and the SCOOP}

The discussion of uniqueness in Section 5 and the examples in the previous section suggest that there is a relationship between the core and the SCOOP. These discussions also advanced the nature of the relationship of the SCOOP with one particular selection of the core, the CNBS proposed by Compte and Jehiel (2010). In this section, we examine these relationships more in depth in the context of superadditive games. ${ }^{12}$

The definition of a SCOOP implies that if the solution is deterministic then the payoffs must be an imputation in the core. Indeed, in a deterministic SCOOP each player's disagreement payoff in any non winning coalition equals the payoff she obtains in the grand (winning) coalition, $t_{i}^{s}=u_{i}^{N}, s \neq N$. Thus, no coalition can have a worth above the sum of its members' payoffs in the grand coalition, $v_{s} \leq \sum_{i \in s} t_{i}^{s}=\sum_{i \in s} u_{i}^{N}$. Otherwise, players would obtain a payoff in that coalition strictly above the one they obtain in the grand coalition, $u_{i}^{s}>t_{i}^{s}=u_{i}^{N}$, which would contradict part (iii) in the definition of the SCOOP. Therefore, the vector of payoffs predicted by a deterministic SCOOP is an imputation in the core. An immediate implication is that, if the core is empty, then the

\footnotetext{
${ }^{12}$ For other classes of games (e.g., three-player, three-cake problem; indivisible games, defined as games where any two coalitions with positive worth must share at least one player; etc.), we may consider a straightforward extension of the core and the CNBS for which the discussion in this section applies.
} 
SCOOP will necessarily be probabilistic.

In addition, the outside-option principle embedded in the definition of the SCOOP places restrictions on the core imputations that can be part of a deterministic SCOOP. In particular, a coalition $s, s \neq N$, may affect the disagreement payoffs, and hence the final payoffs, in the grand coalition only if the sum of disagreement payoffs adds up to exactly the worth of the coalition: $v_{s}=\sum_{i \in s} t_{i}^{s}=\sum_{i \in s} u_{i}^{N}$. Indeed, if $v_{s}<\sum_{i \in s} t_{i}^{s}=\sum_{i \in s} u_{i}^{N}$, then line 2 of part (i) in the definition of the SCOOP would make $u_{i}^{s}=0$, and so would make the coalition irrelevant; that is, $\left\{t_{i}^{N}\right\}_{i \in N}$ would be independent of $v_{s}$. Hence, in a deterministic SCOOP, the players obtain in the winning coalition the same payoff they could also get in the unsuccessful, yet influential, coalitions.

Since players equally share the net surplus of the grand coalition (first line of part (i) in the definition of a SCOOP), $u_{i}^{N}-t_{i}^{N}=u_{j}^{N}-t_{j}^{N}$, for any $i, j$, and since $t_{i}^{N}=\sum_{s \ni i} \lambda_{s}^{N} u_{i}^{N}$, then we have

$$
u_{i}^{N}\left(1-\sum_{s \ni i} \lambda_{s}^{N}\right)=u_{j}^{N}\left(1-\sum_{s \ni i} \lambda_{s}^{N}\right),
$$

(This expression is nothing but the conditional equal loss property of the SCOOP that we mentioned in the previous section, and that we formally derive in the proof of Theorem 2.)

These observations may be used as the starting point to explain the relationship of our theory with the theory of coalition formation proposed by Compte and Jehiel (2010) for games with a non-empty core. They define a solution concept, the CNBS, as that imputation in the core that maximizes the Nash product:

$$
\begin{array}{ll} 
& \max _{x \in \mathbb{R}_{+}^{n}} \Pi_{i \in N} x_{i} \\
\text { s.t. } \quad \sum_{i \in N} x_{i} \leq v_{N}, \\
\sum_{i \in s} x_{i} \geq v_{s}, \forall s \neq N .
\end{array}
$$

For games with a core with a non-empty interior, the solution to this problem satisfies the first order conditions

$$
\begin{aligned}
\Pi_{i \in N} x_{i} & =x_{i}\left(\phi-\sum_{s \ni i} \delta_{s}\right), \quad \forall i, \\
\delta_{s}\left(\sum_{i \in s} x_{i}-v_{s}\right) & =0, \forall s,
\end{aligned}
$$

where $\delta_{s}$ is the Lagrange multiplier of the restriction in (2) associated to coalition $s$, and $\phi$ is the one corresponding to the grand coalition $N$. Now, dividing by $\phi>0$ the first 
line of (3) corresponding to players $i$ and $j$, we obtain (1) with only setting $x_{i}=u_{i}^{N}$ and $\lambda_{s}^{N}=\delta_{s} / \phi$. Obviously, the system of equalities formed by the binding constraints must also be satisfied by both solutions. Thus, a deterministic SCOOP solves problem (2) and so coincides with the CNBS. Put in other words, maximizing the Nash product in (2) is homomorphic to equally sharing the surplus (net of disagreement payoffs) in $N$ given the counterfactuals as defined in the SCOOP.

Thus, the relative strength of coalition $s$ (as discussed in Compte and Jehiel, 2010) is measured by the ratio $\delta_{s} / \phi$. In the context of the SCOOP, such a ratio has a precise interpretation as the probability of success of that coalition in case of a breakup of negotiations in the grand coalition. ${ }^{13}$ This interpretation also explains why the SCOOP and the CNBS do not necessarily coincide in games with a non-empty core interior, for which both concepts are well defined. Indeed, the beliefs $\left\{\lambda_{s}^{N}\right\}$ have another constraint to meet: its components associated to binding coalitions in the solution to (2) cannot add to more than 1 (they are part of a probability distribution). ${ }^{14}$ The latter is a constraint that $\left\{\delta_{s} / \phi\right\}$ may not satisfy, in which case the CNBS is not the SCOOP. Put in other words, if $\sum_{s} \frac{\delta_{s}}{\phi}>1$ then there are no consistent counterfactuals that sustain the CNBS. In that case, no deterministic SCOOP exists, and so our solution predicts a probabilistic outcome even though the core is not empty.

Compte and Jehiel (2010) call $\phi-\sum_{s} \delta_{s} \geq 0$ Property P1. ${ }^{15}$ Necessary and sufficient conditions for an efficient SCOOP are easier to state if we disregard the borderline cases, including $\phi-\sum_{s} \delta_{s}=0$, when multiple SCOOPs exist:

Remark When the SCOOP is unique (which is generically the case), then the CNBS and the SCOOP coincide if and only if the core of the game has a non-empty interior and $\phi-\sum_{s} \delta_{s}>0$ in the solution to problem (2). In this case, and only in this case, the unique SCOOP is deterministic and so efficient. ${ }^{16}$

\footnotetext{
${ }^{13}$ The ratio $\frac{\delta_{s}}{\phi}$ in (3) measures the - per unit - increase in $v_{N}$ needed to leave the maximum Nash product unchanged when $v_{s}$ increases in one unit. The value $\lambda_{s}^{N}$ in the SCOOP is the increase in claims that players in $s$ bring to the grand coalition. Thus, it also measures the increase of $v_{N}$ needed to leave unchanged the net surplus in the grand coalition. As we mentioned above, $\frac{\delta_{s}}{\phi}$ and $\lambda_{s}^{N}$ coincide in the (interior of ) the region where the SCOOP is deterministic.

${ }^{14}$ There is always at least one coalition that is not binding in problem (2); for instance, individual coalitions. Hence, the sum of $\lambda_{s}^{N}$, for $s$ binding, can (and generically will) be strictly less than one.

${ }^{15}$ One of the key results in Compte and Jehiel (2010) is that the limiting equilibrium of their noncooperative bargaining protocol (random proponent) is efficient if and only if the game satisfies Property P1.

${ }^{16}$ If the core of the game has a non-empty interior and $\sum_{s} \frac{\delta_{s}}{\phi}=1$, then a deterministic SCOOP still exists and coincides with the CNBS. However, there are other probabilistic SCOOPs. Also, there are
} 


\section{Computing the SCOOP}

The SCOOP of a game can be easily computed following the characterization discussed in Section 6 and formally proved in the Appendix (Lemmas 7 and 6). In the case of games with a relatively small number of players and/or small number of relevant coalitions, computing the SCOOP is straightforward. More generally, we can use the following algorithm, which can be easily programmed with a numerical software. ${ }^{17}$

1) Looking for a deterministic SCOOP.

1.a: For each subset $Z$ of coalitions in $S-\{N\}$, obtain the solution $\left(\lambda^{N}, u, \widetilde{\omega}\right)$, where $\lambda^{N} \in \mathbb{R}^{\# Z}, u \in \mathbb{R}^{n}$ and $\widetilde{\omega} \in \mathbb{R}$ to the system

$$
\begin{aligned}
\left(1-\sum_{s \ni i, s \in Z} \lambda_{s}^{N}\right) u_{i} & =\widetilde{\omega}, \forall i \\
\sum_{i \in s} u_{i} & =v_{s}, \quad \forall s \in Z \cup\{N\} .
\end{aligned}
$$

1.b: Check that $\widetilde{\omega}, \lambda_{s}^{N}, u_{i} \geq 0, \sum_{s \in Z} \lambda_{s}^{N} \leq 1$, and $\sum_{i \in s} u_{i} \geq v_{s}$ for all $s \notin Z$.

1.c: If success, stop; otherwise, return to 1.a with a different set $Z$ if there is at least one left; otherwise, go to 2.

2) Looking for a non-deterministic SCOOP.

2.a: For each subset $Z$ of coalitions in $S$, define $I$ as the set of players that belong to at least one coalition in $Z$, and obtain the solution $(p, u, \omega)$, where $p \in \mathbb{R}^{\# Z}, u \in \mathbb{R}_{+}^{I}$ and $\omega \in \mathbb{R}_{+}$to the system

$$
\begin{aligned}
\left(1-\sum_{s \ni i, s \in Z} p_{s}\right) u_{i} & =\omega, \quad \forall i \in I, \\
\sum_{i \in s} u_{i} & =v_{s}, \quad \forall s \in Z, \\
\sum_{s \in Z} p_{s} & =1 .
\end{aligned}
$$

2.b: Check that $\omega, p_{s}, u_{i} \geq 0$, and if $i \notin I$, impute $u_{i}=\omega$, and check $\sum_{i \in s} u_{i} \geq v_{s}$ for all $s \notin Z$.

2.c: If success, stop; otherwise, return to 2 .a with a different set $Z$.

Our results imply that there is a (generically unique) solution to this algorithm when the game is superadditive. If the game is not superadditive, the algorithm may still be games with a non-empty core but an empty core interior that also have a deterministic SCOOP.

${ }^{17}$ Stage 1 in this algorithm is simply the algorithm to solve the Kuhn-Tucker conditions for the optimization problem that defines the CNBS. 
used substituting any efficient coalition for $\mathrm{N}$ in 1). In that case, there is no guarantee that the solution to the algorithm is generically unique.

\section{Concluding remarks}

In this paper we have proposed a new solution concept, the SCOOP, for games in characteristic function form that can be interpreted as a generalization of the NBS for coalition formation problems. The SCOOP is based on the idea of simultaneous — virtual - negotiations in all possible coalitions. In each coalition players negotiate à la Nash with endogenous disagreement points based on consistent beliefs about the implications of failure in that particular negotiation. The requirement of consistent beliefs is the most salient feature of our concept and probably our main contribution. We show that the SCOOP always exists, is generically unique for superadditive games, and easy to compute. Moreover, we identify the set of games for which the outcome is probabilistic and hence inefficient. Thus, our concept stands ready to be applied to old and new coalition formation problems in economics and other social sciences.

Our discussion of uniqueness in Section 4 restricted attention to superadditive games. Superadditivity is a sufficient but not a necessary condition for (generic) uniqueness. In fact, to show uniqueness all we need is that, within the set of relevant coalitions (those with a positive probability of success), any pair of coalitions must be connected by a chain in which a coalition shares at least one player with the preceding and with the subsequent coalition. This property is typically satisfied in superadditive games, as disjoint coalitions are dominated by larger coalitions. But it can also be ascertained for other special classes of games that are not necessarily superadditive. In particular, games in which any pair of coalitions with positive worth share at least one player generically possess a unique SCOOP which, as in the case of superadditive games, may or may not be efficient. ${ }^{18}$ Another class of games with unique SCOOP is that of symmetric games, in which the value of the coalition only depends on its size. For any symmetric game, the unique SCOOP (in payoffs and individual probabilities of success) assigns equal probabilities to all the coalitions with the maximal value per player (notice that the set of multiple-

\footnotetext{
${ }^{18} \mathrm{~A}$ popular example of an indivisible game is the three-player, three-cake problem (a game in which only the bilateral coalitions generate a positive value). An extreme form of indivisibility are games with essential players. That is, the intersection of all positive-value coalitions is non-empty. The SCOOP of these games is necessarily deterministic since they have a non-empty core and Property P1 is satisfied: the first order condition that characterizes the CNBS for an essential player implies that $\sum_{s} \frac{\delta_{s}}{\phi}<1$.
} 
player coalitions of the same size is connected), and players equally share the worth of the coalition. ${ }^{19}$ Thus, once again, the SCOOP of a symmetric game may or may not be efficient, as the coalition size with the maximal value and with the maximal value per player may not coincide.

The ideas that we have discussed in this paper may be of interest for investigating other classes of games, in particular games where (general types of) externalities are allowed, and also more than one disjoint coalition can form. That is, games in partition function form. For games in partition function form, a similar concept would have to predict a probability distribution, $p$, over partitions of $N$ rather than over coalitions. Disagreement payoffs in one coalition could be computed as payoffs expected in the counterfactual event that the coalition does not form. The main difficulty that such theory would face is defining what is the counterfactual - in terms of partitions - of a coalition - not a partition - forming. We think that this is a promising avenue worth investigating.

\section{References}

Bennett, E. (1997), "Multilateral Bargaining Problems." Games and Economic Behavior, 19(2), 151-179.

Binmore, K. (1985), "Bargaining and Coalitions." Chapter 13 in Game-Theoretic Models of Bargaining, Alvin Roth (editor). Cambridge: Cambridge University Press, 269-304.

Chatterjee, K., B. Dutta, D. Ray, and K. Sengupta (1993), "A Noncooperative Theory of Coalitional Bargaining." Review of Economic Studies, 60, 463-470.

Compte, O. and P. Jehiel (2010), "The Coalitional Nash Bargaining Solution." Econometrica, 78(5), 1593-1623.

Hart, S. and A. Mas-Colell (1989), "Potential Value and Consistency." Econometrica $57(3), 589-614$.

Highham, J. (1990), "Analysis of the Cholesky decomposition of a semi-definite matrix," in Reliable Numerical Computation, M. G. Cox and S. Hammarling, eds., Oxford University Press, pp. 161-185.

Owen, G. (1995), Game Theory. Academic Press.

Peleg, B. (1986), "On the Reduced Game Property and its Converse", International Journal of Game Theory 15(3), 187-200.

\footnotetext{
${ }^{19} \mathrm{~A}$ formal proof of this claim is available upon request.
} 
Serrano, R., and K.I.Shimomura (1998), "Beyond Nash Bargaining Theory: The Nash Set", Journal of Economic Theory, 83(2), 286-307.

Sobolev, A. I. (1975), "A characterization of optimality principles in cooperative games by functional equations." Mathematical Methods in the Social Sciences 6, 94-151 (in Russian).

\section{Appendix}

\subsection{Existence: Proof of Theorem 1}

Given a vector $\mathbf{p}=\left\{p_{s}\right\}_{s \in S}$, consider the equation

$$
\mathbf{B} \cdot \mathbf{x}=-\mathbf{p} \cdot \mathbf{v} \cdot \mathbf{1}
$$

where $\mathbf{1}$ is the vector of dimension $n$ with all components equal to $1, \mathbf{v}=\left\{v_{s}\right\}_{s \in S}$ and $\mathbf{B}$ is the $n \times n$ matrix whose element $b_{i j}=-1$ if $i \neq j$ and

$$
b_{i i}=n\left(1-q_{i}\right)-1,
$$

where $q_{i}=\sum_{s \ni i} p_{s}$. Let $\Delta^{\varepsilon}$ denote the convex, compact set

$$
\Delta^{\varepsilon}=\left\{\mathbf{p} \in \Delta: p_{r} \geq \varepsilon \forall r \in S\right\}
$$

where $\Delta$ is the simplex in $\mathbb{R}^{|S|}$. Also, let $\Delta^{\cup}=\cup_{\varepsilon>0} \Delta^{\varepsilon}$, and let

$$
\Delta^{q}=\left\{\mathbf{p} \in \Delta: q_{i}<1 \forall i \in N\right\}
$$

Note that $\Delta^{\cup} \subset \Delta^{q}$. We first prove the following lemma.

Lemma $4 \mathbf{B}$ is invertible on $\Delta^{q}$. Thus, (4) defines $\mathbf{x}(\mathbf{p})$ as an implicit, linear differentiable function on $\Delta^{q}$.

Proof. Substract the last row of $\mathbf{B}$ from all other rows of the matrix. The resulting matrix has zeros in all components of the rows 1 through $n-1$ except in the diagonal and in the last column. In row $i$, the diagonal element is $b_{i i}+1$, and the element in the last column $-\left(b_{n n}+1\right)$. Now multiply each row $i$, from 1 through $n-1$, by $\frac{1}{b_{i i}+1}$ and add all of them to row $n$. We then have a triangular matrix (all components below the 
diagonal are zeros). Thus, the eigenvalues of this matrix are the elements of the diagonal: $b_{i i}+1=n\left(1-q_{i}\right)$ for each $i<n$ and

$$
b_{n n}-\left(b_{n n}+1\right)\left[\sum_{i \neq n} \frac{1}{b_{i i}+1}\right] .
$$

This eigenvalue is also nonzero. Indeed, we can write (5) as

$$
\begin{aligned}
& \left(b_{n n}+1\right)\left[\frac{\prod_{i=1}^{n}\left(b_{i i}+1\right)-\sum_{i=1}^{n} \prod_{j \neq i}\left(b_{j j}+1\right)}{\prod_{i=1}^{n}\left(b_{i i}+1\right)}\right] \\
= & -\left(1-q_{n}\right) \sum_{i=1}^{n} \frac{q_{i}}{1-q_{i}}<0
\end{aligned}
$$

Since this matrix is obtained by row operations on $\mathbf{B}$, we conclude that $\mathbf{B}$ also has a nonzero determinant.

Note that $x_{i}(\mathbf{p})\left(1-q_{i}\right)$ is the same for all players, and equals

$$
\frac{\sum_{j} x_{j}(\mathbf{p})-\mathbf{p} \cdot \mathbf{v}}{n}
$$

Also, we construct another function, $\mathbf{z}: \Delta^{q} \rightarrow \mathbb{R}^{|S|}$, based on $\mathbf{x}$ and defined as $z_{s}(\mathbf{p})=$ $\sum_{i \in s} x_{i}(\mathbf{p})-v_{s} \cdot \mathbf{z}(\mathbf{p})$ satisfies an important property: for any $\mathbf{p} \in \Delta^{q}, \mathbf{p} \cdot \mathbf{z}(\mathbf{p})=0$. Indeed, adding the $n$ equations in (4),

$$
\sum_{s \in S} p_{s}\left(\sum_{i \in s} x_{i}(\mathbf{p})-v_{s}\right)=\sum_{i \in N} q_{i} x_{i}(\mathbf{p})-\mathbf{p} \cdot \mathbf{v}=0 .
$$

As a consequence, we cannot have that $z_{s}(\mathbf{p})>0$ for all $s \in S$. Also, by the same argument, we cannot have that $\mathbf{p}$ puts positive weight only on $s$ such that $z_{s}(\mathbf{p})<0$.

To complete the definition of $\mathbf{x}(\mathbf{p})$ and $\mathbf{z}(\mathbf{p})$ for the case $\mathbf{p} \in \Delta-\Delta^{q}$, consider any sequence $\{\mathbf{p}(l)\} \rightarrow \mathbf{p}$, and define $\mathbf{x}(\mathbf{p})=\lim _{l} \mathbf{x}(\mathbf{p}(l))$ and $\mathbf{z}(\mathbf{p})=\lim _{l} \mathbf{z}(\mathbf{p}(l))$. Note that every point of $\Delta$ is a limit point of $\Delta^{q}$-i.e., $\Delta^{q}$ is dense in $\Delta$ - and $x$ is (linear and so) uniformly continuous, so this continuous extension is well defined (unique), by the Continuous Extension Theorem. Also, note that $\mathbf{p} \cdot \mathbf{z}(\mathbf{p})=0$ even for $\mathbf{p} \in \Delta-\Delta^{q}$.

Using these functions, and for arbitrary, given $\varepsilon>0$, we construct a correspondence $h: \Delta^{\varepsilon} \rightarrow \Delta^{\varepsilon}$ as follows:

$$
h_{\varepsilon}(\mathbf{p})=\left\{\widetilde{\mathbf{p}} \in \Delta^{\varepsilon}: \widetilde{\mathbf{p}} \in \arg \min _{\overline{\mathbf{p}} \in \Delta^{\varepsilon}} \overline{\mathbf{p}} \mathbf{z}(\mathbf{p})\right\} .
$$

Note that $\overline{\mathbf{p}} \mathbf{z}(\mathbf{p})$ is a linear function of $\overline{\mathbf{p}}$ and so $h_{\varepsilon}(\mathbf{p})$ is non-empty and convex. Finally, $\mathbf{z}$ is continuous, and then trivially $h_{\varepsilon}$ is upper hemi-continuous. Thus, from Kakutani's fixed point theorem, we conclude that $h_{\varepsilon}$ has a fixed point in $\Delta^{\varepsilon}$. 
Some properties of these (limits of) fixed points, are important here. Consider a sequence $\{\varepsilon(l)\} \rightarrow 0$ and a corresponding, converging sequence of fixed points $p(l)$ for the correspondence $h_{\varepsilon(l)}$. The sequence

$$
\lambda_{r}^{s}(l)=\frac{p(l)_{r}}{1-p(l)_{s}} \in[0,1]
$$

$\lambda^{s}(l)$ is also a sequence in a compact set, $[0,1]^{|S|-1}$, for all $s$. Then, the sequence $p(l)$ contains a subsequence that converges and where $\lambda^{s}(l)$ also converges for all $s$. Note that the limit $\lambda_{r}^{s} \geq 0$ and since $\sum_{r \neq s} \lambda_{r}^{s}(l)=1$, then

$$
\sum_{r \neq s} \lambda_{r}^{s}=\lim _{l} \sum_{r \neq s} \lambda_{r}^{s}(l)=1 .
$$

Moreover

Lemma $5 \mathbf{z}(\mathbf{p}) \geq 0$.

Proof. Assume otherwise. That is, assume that $z_{s}(\mathbf{p})=\beta<0$. The continuity of $\mathbf{z}$ implies that for $l$ large, $z_{s}(\mathbf{p}(l))<\beta / 2$. Thus, $p_{r}(l)=\varepsilon(l)$ for all $r$ such that $z_{r}(\mathbf{p}(l))>$ $\beta / 2$. Therefore, in the limit, $\mathbf{p} \cdot \mathbf{z}(\mathbf{p})<0$, which contradicts the fact that, for all $\mathbf{p}$, $\mathbf{p} \cdot \mathbf{z}(\mathbf{p})=0$.

Given those limits $\mathbf{p}$ and $\boldsymbol{\lambda}^{s}$, we now construct a candidate SCOOP as the four-tuple $\left\{\left\{u_{i}^{s}\right\}_{i \in s},\left\{t_{i}^{s}\right\}_{i \in s}, p_{s},\left\{\lambda_{r}^{s}\right\}_{r \neq s}\right\}_{s \in S}$ where:

1) $p_{s}$ are the components of the vector $\mathbf{p}$;

2) $\lambda_{r}^{s}$ are the components of $\boldsymbol{\lambda}^{s}$;

3) If $p_{s}>0$, then $u_{i}^{s}=x_{i}(\mathbf{p})$. If $p_{s}=0$, then $u_{i}^{s}$ is defined according to i) in the definition of a SCOOP for these threat points.

4) $t_{i}^{s}=\sum_{r \ni i, r \neq s} \lambda_{r}^{s} u_{i}^{r}$, where $u_{i}^{r}$ and $\lambda_{r}^{s}$ are as defined in 2) and 3).

It is important to notice that, given the definition of $h_{\varepsilon}$, whenever $z_{r}(p)>0, \lambda_{r}^{s}(l)=$ $\varepsilon(l)$ for sufficiently large $l$, and so $\lambda_{r}^{s}=0$. Likewise, $\lambda_{r}^{s}>0$ only if $z_{r}(\mathbf{p})=0$, in which case $u_{i}^{r}=x_{i}(\mathbf{p})$. Also, even if $p_{s}=1, \sum_{r \neq s} \lambda_{r}^{s}=1$.

We show that this four-tuple satisfies the definition of a SCOOP. First, from (6) and 2), $\lambda_{r}^{s}=\frac{p_{r}}{1-p_{s}}$ when $p_{s}<1$ as required in (ii). Also, if $p>0$, we have defined $t_{i}^{s}=\sum_{r \ni i, r \neq s} \lambda_{r}^{s} u_{i}^{r}$. Finally, $p_{r} x_{i}(\mathbf{p})=0$ when $p_{r}=0$, and $p_{r} x_{i}(\mathbf{p})=p_{r} u_{i}^{r}$ if $p_{r}>0$. Moreover, if $p_{s}=0$ then $\lambda_{r}^{s}=p_{r}$, and so $\sum_{r \ni i, r \neq s} \lambda_{r}^{s} u_{i}^{r}=q_{i} x_{i}(\mathbf{p})$. Thus, (ii) is satisfied.

That also implies that, from 3), (i) is satisfied for $s$ such that $p_{s}=0$. For $s$ such that $p_{s} \in(0,1)$ (and so $\left.z_{s}(\mathbf{p})=0\right)$, we have that $\left(1-q_{i}\right) u_{i}^{s}=\left(1-q_{j}\right) u_{j}^{s}$ for all $i, j \in s$, and so, 
dividing both sides of the equality by $\left(1-p_{s}\right)$ and simplifying, taking into account that $\lambda_{r}^{s}=\frac{p_{r}}{1-p_{s}}$ in this case and $u_{i}^{s}=u_{i}^{r}$ for all $r$ with positive probability, we have

$$
u_{i}^{s}-\sum_{r \ni i, r \neq s} \lambda_{r}^{s} u_{i}^{r}=u_{j}^{s}-\sum_{r \ni j, r \neq s} \lambda_{r}^{s} u_{j}^{r}
$$

which together with $z_{s}(\mathbf{p})=0$ implies (i). Therefore, it only remains to check that (i) is satisfied for $s$ such that $p_{s}=1$. Note that, in this case, $u_{i}^{r}>0$ only if $z_{r}(\mathbf{p})=0$, and in these cases $u_{i}^{r}=x_{i}(\mathbf{p})$ according to 3$)$. Then, according to 4$), t_{i}^{s}=\sum_{r \ni i, r \neq s} \lambda_{r}^{s} x_{i}(\mathbf{p})$, and so $u_{i}^{s}-t_{i}^{s}=x_{i}(\mathbf{p})\left(1-\sum_{r \ni i, r \neq s} \lambda_{r}^{s}\right)$. But,

$$
\begin{aligned}
& x_{i}(\mathbf{p}(l))\left(1-q_{i}(l)\right)=x_{i}(\mathbf{p}(l))\left(1-\sum_{r \ni i} p_{r}(l)\right) \\
= & x_{i}(\mathbf{p}(l))\left(1-p(l)_{s}\right)\left(1-\sum_{r \ni i, r \neq s} \frac{p(l)_{r}}{1-p(l)_{s}}\right) \\
= & \left(1-p(l)_{s}\right) x_{i}(\mathbf{p}(l))\left(1-\sum_{r \ni i, r \neq s} \lambda_{r}^{s}(l)\right)
\end{aligned}
$$

Thus, since $x_{i}(\mathbf{p}(l))\left(1-q_{i}(l)\right)$ is common to all players, $x_{i}(\mathbf{p}(l))\left(1-\sum_{r \ni i, r \neq s} \lambda_{r}^{s}(l)\right)$ is also common to all players in $s$, and so this is also the case in the limit. Therefore, $u_{i}^{s}-t_{i}^{s}=u_{j}^{s}-t_{j}^{s} \geq 0$, for all $i, j \in s$, which, again, together with $z_{s}(\mathbf{p})=0$ implies (i).

Turning to (iii), we need only show that if $p_{r}=0$ then $u_{i}^{r} \leq x_{i}(\mathbf{p})$. Note that, if $p_{r}=0$, then

$$
u_{i}^{r}=q_{i} x_{i}(\mathbf{p})+\frac{1}{n_{r}}\left(v_{r}-\sum_{j \in r} q_{j} x_{j}(\mathbf{p})\right),
$$

so that, adding and substracting $\frac{1}{n_{r}} \sum_{j \in r} x_{j}(\mathbf{p}), x_{i}(\mathbf{p})-u_{i}^{r}$ is

$$
\left(1-q_{i}\right) x_{i}(\mathbf{p})-\frac{1}{n_{r}}\left(v_{r}-\sum_{j \in r} x_{j}(\mathbf{p})+\sum_{j \in r}\left(1-q_{j}\right) x_{j}(\mathbf{p})\right) \geq 0,
$$

where the inequality follows from $z_{r}(\mathbf{p}) \geq 0$, and again the fact that $\left(1-q_{i}\right) x_{i}(\mathbf{p})=(1-$ $\left.q_{j}\right) x_{j}(\mathbf{p})$ for all $i, j$.

\subsection{Uniqueness: Proof of Theorem 2}

We now prove Theorem 2, the generic uniqueness of the SCOOP for superadditive games. For that purpose, we begin by formally stating and proving certain properties. As in the proof of Theorem 1 , let $q_{i}=\sum_{s \ni i} p_{s}$ for all $i \in N$. 
Lemma $6 \sigma=\left\{\left\{u_{i}^{s}\right\}_{i \in s},\left\{t_{i}^{s}\right\}_{i \in s}, p_{s},\left\{\lambda_{r}^{s}\right\}_{r \neq s}\right\}_{s \in S}$ is a non-deterministic SCOOP only if it satisfies: (a) $u_{i}^{s}=u_{i}$ for some $u_{i}$, for all $s \in S_{+}(\sigma)$ and for all $i \in \cup S_{+}(\sigma)$, where $S_{+}(\sigma)=\left\{s \in S\right.$ s.t. $\left.p_{s}>0\right\}$. (b) $\sum_{j \in s} u_{j}-v_{s}=0$ for all $s \in S_{+}(\sigma)$. (c) There exists $\omega \geq 0$ such that $\left(1-q_{i}\right) u_{i}=\omega$, for all $i \in \cup S_{+}(\sigma)$. (Equal loss property.) In fact, $\omega>0$ unless $v_{s}=v_{N} \forall s \in S_{+}(\sigma)$. (d) Let $u_{k}=\omega, \forall k \notin \cup S_{+}(\sigma)$; then $\sum_{j \in s} u_{j}-v_{s} \geq 0$ for all $s \notin S_{+}(\sigma)$.

Proof. From part (iii) in the definition of $S C O O P$, any player that is in more than one coalition in $S_{+}(\sigma)$ must be indifferent between them. Hence, $u_{i}^{s}=u_{i}$ for all $i \in s$ and all $s \in S_{+}(\sigma)$. This is point (a). Suppose that $v_{s}-\sum_{i \in s} t_{i}<0$ for some $s \in S_{+}(\sigma)$, so that $u_{i}^{s}=0$ for all $i \in s$. From part (a), this implies that $u_{i}=0$ for all players in $s$, and so $\sum_{i \in s} t_{i}=0$ which contradicts $v_{s}-\sum_{i \in s} t_{i}<0$. Thus, $v_{s}-\sum_{i \in s} t_{i} \geq 0$, and so from (i) in the definition of a SCOOP, $\sum_{j \in s} u_{j}=v_{s}=0$, which is point (b).

Consider a coalition $s$ such that $v_{s}-\sum_{i \in s} t_{i} \geq 0$. Since $t_{i}^{s}=\frac{q_{i}-p_{s}}{1-p_{s}} u_{i}$ for all $i \in s$, then from (i) in the definition of a SCOOP we can write

$$
u_{i}^{s}=\frac{q_{i}-p_{s}}{1-p_{s}} u_{i}+\frac{1}{n_{s}}\left(v_{s}-\sum_{j \in s} \frac{q_{j}-p_{s}}{1-p_{s}} u_{j}\right),
$$

which, using $\sum_{j \in s} u_{j}=v_{s}$ from (b), is equivalent to $u_{i}^{s}=q_{i} u_{i}+\omega_{s}$, where

$$
\omega_{s} \equiv \frac{1}{n_{s}}\left(v_{s}-\sum_{j \in s} q_{j} u_{j}\right) .
$$

That is, from (a), $\left(1-q_{i}\right) u_{i}=\omega_{s}$. The left hand side is independent of $s$. Thus, for any two coalitions $s, s^{\prime}$ such that $s \cap s^{\prime} \neq \varnothing, \omega_{s}=\omega_{s^{\prime}}$. Now, suppose that two coalitions $s, s^{\prime} \in S_{+}(\sigma)$ are such that $s \cap s^{\prime}=\varnothing$. If $s \cup s^{\prime} \in S_{+}(\sigma)$, then by the argument above, $\omega_{s}=\omega_{s^{\prime} \cup s}=\omega_{s^{\prime}}$. Thus, suppose that $s \cup s^{\prime} \notin S_{+}(\sigma)$. Then, $v_{s_{1} \cup s_{2}}-\sum_{i \in s_{1} \cup s_{2}} t_{i}^{s_{1} \cup s_{2}} \geq$ $v_{s_{1}}+v_{s 2}-\sum_{i \in s_{1} \cup s_{2}} q_{i} u_{i}=\sum_{i \in s_{1} \cup s_{2}}\left(1-q_{i}\right) u_{i} \geq 0$. The first inequality uses superadditivity plus the definition of disagreement points for a coalition outside $S_{+}$. The second equality simply uses the fact that the sum of payoffs in coalitions $s_{1}$ and $s_{2}$ are equal to the value of these coalitions. Therefore, for all $i \in s \cup s^{\prime}, u_{i}^{s \cup s^{\prime}}=q_{i} u_{i}+\omega_{s \cup s^{\prime}}$. Also, by superadditivity $\omega_{s \cup s^{\prime}} \geq \frac{n_{s}}{n_{s}+n_{s^{\prime}}} \omega_{s}+\frac{n_{s^{\prime}}}{n_{s}+n_{s^{\prime}}} \omega_{s^{\prime}}$. Without loss of generality, suppose that $\omega_{s}>\omega_{s^{\prime}}$. Then, $\omega_{s \cup s^{\prime}}>\omega_{s^{\prime}}$, which implies that for all $i \in s, u_{i}^{s}=u_{i}<u_{i}^{s \cup s^{\prime}}$, which contradicts part (iii) of the definition of a SCOOP. Therefore, if the game is superadditive even if coalitions in $S_{+}(\sigma)$ are disjoint they still must have the same $\omega$, and hence the equal loss property of part (c) must hold. 
Consider now a coalition $s \notin S_{+}(\sigma)$. If $v_{s}-\sum_{i \in s} t_{i}^{s}<0$, since $t_{i}^{s}=q_{i} u_{i} \leq u_{i}$, then $v_{s}-\sum_{i \in s} u_{i}<0$. If instead $v_{s}-\sum_{i \in s} t_{i}^{s} \geq 0$, then as mentioned above for all $i \in s$, $u_{i}^{s}=q_{i} u_{i}+\omega_{s}$. From part (iii) of the definition of SCOOP, $u_{i}^{s} \leq u_{i}$ for all $i \in \cup S_{+}(\sigma)$. Hence,

$$
\left(1-q_{i}\right) u_{i} \geq \omega_{s}
$$

Also, let $u_{k}=\omega$ obtained above for every player $k \notin \cup S_{+}(\sigma)$, i.e., so that $q_{k}=0$. Thus, adding (8) for all $i \in s$,

$$
\sum_{i \in s}\left(1-q_{i}\right) u_{i} \geq n_{s} \omega_{s}=v_{s}-\sum_{i \in s} q_{i} u_{i}
$$

or, equivalently,

$$
\sum_{i \in s} u_{i} \geq v_{s}
$$

This is point (d). Finally, we show that $\omega>0$ unless there is more than one efficient coalition. Indeed, suppose $\omega=0$. This implies that $q_{i}=1$ for all $i \in \cup S_{+}(\sigma)$ such that $u_{i}>0$. That is, $u_{i}>0$ only for players in $\cap S_{+}(\sigma)$. For all $r \in S_{+}(\sigma)$, and from (b),

$$
v_{r}=\sum_{i \in r} u_{i}=\sum_{i \in N} u_{i} \geq v_{N},
$$

which implies that $v_{r}=v_{N}$. This completes the proof of point (c).

Let $r$ be the coalition that succeeds with probability one in a deterministic SCOOP. In this case Bayes rule does not apply and we can freely choose the conditional probabilities $\left\{\lambda_{s}^{r}\right\}_{s \neq r}$. We denote by $S_{0}(\sigma)$ the set of coalitions with positive worth for which there is an agreement; i.e., $S_{0}(\sigma)=\left\{s \in S\right.$, s.t. $\left.\sum_{i \in s} u_{i}^{s}=v_{s}>0\right\}$. Also, let $\widetilde{q}_{i}=\sum_{s \in S_{0}(\sigma), s \ni i} \lambda_{s}^{r}$. That is, $\widetilde{q}_{i}$ is the probability that, conditional on the failure of coalition $r$, player $i$ obtains a positive payoff. Since $\lambda_{s}^{r}$ can be strictly positive for $s \notin S_{0}(\sigma)$ (there is always at least one coalition outside $S_{0}(\sigma)$; for instance, individual coalitions), then $\sum_{s \in S_{0}(\sigma)} \lambda_{s}^{r}$ can (and generically will) be strictly less than one.

Lemma $7 \sigma=\left\{\left\{u_{i}^{s}\right\}_{i \in s},\left\{t_{i}^{s}\right\}_{i \in s}, p_{s},\left\{\lambda_{r}^{s}\right\}_{r \neq s}\right\}_{s \in S}$ is a deterministic SCOOP, $p_{r}=1$, only if: (a) Coalition $r$ is an efficient coalition. (b) For all $s \in S_{0}(\sigma)$ and $i \in s, u_{i}^{s}=u_{i}$ for some $u_{i}$. Moreover, $u_{i}^{s}=0$ if $i \notin r$. (c) For all $s \notin S_{0}(\sigma), \sum_{j \in s \cap r} u_{j}-v_{s}>0$, and $u_{i}^{s}=0$. (d) There exists $\widetilde{\omega} \geq 0$ such that $\left(1-\widetilde{q}_{i}\right) u_{i}=\widetilde{\omega}$ for all $i \in r$. (Conditional equal loss property.) In fact, $\widetilde{\omega}>0$, unless $s \in S_{0}(\sigma)$ implies $v_{s}=v_{r}$. 
Proof. If $p_{r}=1$, then for all $s \neq r, t_{i}^{s}=u_{i}^{r}$ if $i \in r \cap s$, and $t_{i}^{s}=0$ otherwise. Also, $\sum_{i \in r} t_{i}^{r} \leq v_{r}$. Indeed, otherwise $u_{i}^{r}=0$ and $t_{i}^{s}=0$ for all $s$, so that $u_{i}^{s}=v_{s} / n_{s}>0=u_{i}^{r}$ for $s$ such that $v_{s}>0$, which contradicts part (iii) of the definition of a SCOOP. Hence, $\sum_{i \in r} u_{i}^{r}=v_{r}$, from (i) of the definition of a SCOOP. Let $u_{i}=u_{i}^{r}$ for all $i \in r$. If $\sum_{j \in s} t_{j}^{s}\left(=\sum_{j \in s \cap r} u_{j}\right)<v_{s}$, then (from part (i) of the definition of a SCOOP) for all $i \in s \cap r, u_{i}^{s}>u_{i}$, which contradicts part (iii) of the definition of a SCOOP. Hence, $\sum_{j \in s \cap r} u_{j}-v_{s} \geq 0$ for all $s$. Also, if $s \neq r$ and $\sum_{j \in s} t_{j}^{s}\left(=\sum_{j \in s \cap r} u_{j}\right)=v_{s}$, (i.e., $\left.s \in S_{0}(\sigma)\right)$, and from (i) in the definition of a SCOOP, then $\left(t_{j}^{s}=\right) u_{j}^{s}=u_{j}$ if $j \in s \cap r$ and $\left(t_{j}^{s}=\right) u_{j}^{s}=0$ if $j \notin r$, and this concludes the proof of (b). If $s \notin S_{0}(\sigma)$, i.e., $\sum_{j \in s \cap r} u_{j}-v_{s}>0$, and since $\sum_{i \in s} t_{i}^{s}=\sum_{i \in s \cap r} u_{i}^{r}$, then from part (i) of the definition of a SCOOP, $u_{i}^{s}=0$ for all $i$, which proves (c). Also,

$$
v_{r}=\sum_{i \in r} u_{i}^{r} \geq \sum_{i \in r \cap s} u_{i} \geq v_{s}
$$

for every $s \in S$, and so $r$ is an efficient coalition. This proves (a). Finally, note that, for $i \in r, t_{i}^{r}=\widetilde{q}_{i} u_{i}$. Hence, from part (i) of the definition of a SCOOP

$$
u_{i}=\widetilde{q}_{i} u_{i}+\frac{1}{n_{r}}\left(v_{r}-\sum_{j \in r} \widetilde{q}_{j} u_{j}\right) .
$$

Letting $\widetilde{\omega}=\frac{1}{n_{r}}\left(v_{r}-\sum_{j \in r} \widetilde{q}_{j} u_{j}\right) \geq 0$, we have $\left(1-\widetilde{q}_{i}\right) u_{i}=\widetilde{\omega}$ for all $i \in r$. If $\widetilde{\omega}=0$ then $\widetilde{q}_{i}=1$ for all $i \in r$ such that $u_{i}>0$. Then, for any coalition $s \in S_{0}(\sigma), s \neq r$ we have from (b) that

$$
v_{r}=\sum_{i \in r} u_{i}=\sum_{i \in s \cap r} u_{i}=v_{s} .
$$

That is, any $s \in S_{0}(\sigma)$ is also an efficient coalition. On the other hand, if $S_{0}(\sigma)=\{r\}$, then $t_{i}^{r}=0$ for all $i \in r$, and so $\widetilde{\omega}>0$, since $v_{r}>0$. This completes the proof of $(\mathrm{d})$.

Since games with more than one efficient coalition are non-generic, in the rest of the proof we focus on games with a unique efficient coalition, which for superadditive games is the grand coalition, $N$. Hence, in a deterministic SCOOP we must have $\widetilde{\omega}>0$, and in a non-deterministic one, $\omega>0$.

Next, we will use the parallel between the SCOOP and the solution to problem (2), i.e., the CNBS, to investigate when the SCOOP may or may not be deterministic. Thus, consider the first order conditions for problem (2): 


$$
\begin{array}{rlrl}
\frac{\mathcal{N}}{x_{i}} & =\phi-\sum_{s \ni i} \delta_{s}, & & \\
\phi\left(\sum_{i \in N} x_{i}-v_{N}\right) & =0, & \\
\delta_{s}\left(\sum_{i \in s \cap N} x_{i}-v_{s}\right) & =0, & \forall s \neq N .
\end{array}
$$

Let $M$ be the set of restrictions (coalitions) that satisfy $\sum_{i \in s} x_{i}-v_{s}=0$. The solution $\left\{x_{i}\right\}_{i \in N}$ to this optimization problem exists and is unique, since the objective function is strictly quasiconcave and the constraints are linear. Hence, conditions (9) are both necessary and sufficient when the interior of the core is non empty.

Let Region I denote the set of games characterized by a core with a non-empty interior and $\phi>\sum_{s \neq N} \delta_{s}$ (Property P1 in Compte and Jehiel, 2010, is satisfied with strict equality). Let Region II be the set of games with (i) an empty core, or (ii) a non-empty core and $\phi<\sum_{s \neq N} \delta_{s}$ (Property P1 fails). Notice that the only games left outside Regions I and II are those with (i) a non-empty core with an empty interior, and (ii) a core with a non-empty interior and $\phi-\sum_{s \neq N} \delta_{s}=0$. These regions are well defined due to the following lemmas. Moreover, the set of games not included in Regions I and II has an empty interior.

Lemma 8 Problem (2) is equivalent to an optimization problem with linearly independent, equality constraints. If the choice set in problem (2), and so the core, has a nonempty interior, then $\phi, \delta_{s}$ are unique and differentiable with respect to $v_{N}$ and $v_{s}$ for all $s \neq N$ in the solution to the equivalent problem. Moreover, $d \delta_{s} / d v_{N}<0$ and $d \phi / d v_{N}>0$ for all $s \neq N$.

The lemma will be proved in three steps

Step 1 Problem (2) is equivalent to a (different) optimization program with the same objective function and equality constraints with non-singular Jacobian of the system of active constraints.

Proof. Let the Jacobian of the system of active constraints in one solution to problem (2) be $A$. Obviously, the solution $x^{*}$ to problem (2) is also the solution to an alternative program with only the constraint $N$ and constraints $M$. Suppose that $A$ is not a linearly independent system, so that $A_{s}$, for some $s \in M \cup\{N\}$ can be written as

$$
A_{s}=\sum_{s^{\prime} \in M \cup\{N\}-\{s\}} \alpha_{s^{\prime}} A_{s^{\prime}},
$$


for some vector $\alpha=\left\{\alpha_{s^{\prime}}\right\}_{s^{\prime} \in M \cup\{N\}-\{s\}}$. This means that

$$
x \cdot A_{s}=x \cdot \sum_{s^{\prime} \in M \cup\{N\}-\{s\}} \alpha_{s^{\prime}} A_{s^{\prime}},
$$

for any vector of payoffs $x$. In particular, it is satisfied by the solution $x^{*}$, so that

$$
v_{s}=x^{*} \cdot A_{s}=x^{*} \cdot \sum_{s^{\prime} \in M \cup\{N\}-\{s\}} \alpha_{s^{\prime}} A_{s^{\prime}}=\sum_{s^{\prime} \in M \cup\{N\}-\{s\}} \alpha_{s^{\prime}} v_{s^{\prime}}
$$

Therefore, any vector $x$ that satisfies the constraints corresponding to the coalitions $s^{\prime} \neq s$, i.e., such that $x \cdot A_{s^{\prime}}=v_{s^{\prime}}$ also satisfies

$$
x \cdot \sum_{s^{\prime} \in M \cup\{N\}-\{s\}} \alpha_{s^{\prime}} A_{s^{\prime}}=\sum_{s^{\prime} \in M \cup\{N\}-\{s\}} \alpha_{s^{\prime}} x A_{s^{\prime}}=\sum_{s^{\prime} \in M \cup\{N\}-\{s\}} \alpha_{s^{\prime}} v_{s^{\prime}}=v_{s},
$$

and so satisfies the constraint related to $s$. Thus, the constraint corresponding to coalition $s$ is redundant. We can therefore exclude this coalition from $M \cup\{N\}$, and the new program would still have the same solution $x^{*}$. Repeating this argument, if needed, we conclude that there exists a set $M^{\prime} \cup\{N\}$ of constraints such that the problem defined by (2) with only these equality constraints has $x^{*}$ as a solution and its Jacobian is nonsingular.

Step 2 Let $\left(x^{*}, \phi, \delta\right)$ and $\left(x^{*}, \phi^{\prime}, \delta^{\prime}\right)$ be two solutions to system (9). Then, $\phi-\sum_{s \in S-\{N\}} \delta_{s}$ $=\phi^{\prime}-\sum_{s \in S-\{N\}} \delta_{s}^{\prime}$. Moreover, if Property P1 is satisfied, then both $\phi$ and $\sum_{s \neq N} \delta_{s}$ are unique.

Proof. The active constraints in (9) can be written as

$$
A \cdot\left[\begin{array}{l}
\phi \\
-\delta
\end{array}\right]=\frac{\overrightarrow{\mathcal{N}}}{x^{*}}
$$

where, similarly as before, $A$ is the $|N| \times(|M|+1)$ matrix with component $a_{i s}=1$ if $i \in s$ and 0 otherwise, for $s \in M \cup\{N\}, \delta$ is the $|M|$ dimensional vector with components $\delta_{s}$, and $\frac{\overrightarrow{\mathcal{N}}}{x^{*}}$ is the $|N|$ dimensional vector with components all equal to $\frac{\mathcal{N}}{x_{i}^{*}}$. If $A$ has rank $|M|+1(\leq|N|)$, then the solution, which exists, is unique. Then Step 2 is trivially satisfied. Otherwise, one of the columns in $A$ is a linear combination of the rest. Consider two possible cases. First, if $A_{N}$ is not spanned by the rest of columns of $A$. In this case, it is not spanned by any subset of them, of course, and if the rank of $A$ is smaller than $|M|+1$ it must be because the set of $|M|$ columns other than $A_{N}$ is not a linearly 
independent system. That is, one of these other columns of $A$, call it $A_{r}$, can be obtained as a linear combination of the columns in $A$ other than $A_{r}$ and $A_{N}$. Then, system (10) can be written as

$$
\begin{aligned}
a_{i N} \phi-\sum_{s \neq r} a_{i s} \delta_{s}-\delta_{r}\left(\sum_{s \neq r} a_{i s} \alpha_{s}\right) & =\frac{\mathcal{N}}{x_{i}^{*}} \Longleftrightarrow \\
a_{i N} \phi-\sum_{s \neq r} a_{i s}\left(\delta_{s}+\alpha_{s} \delta_{r}\right) & =\frac{\mathcal{N}}{x_{i}^{*}},
\end{aligned}
$$

for some vector $\alpha \in \mathbb{R}^{|M|-1}$. Thus, $\phi$ and $\widehat{\delta}_{s}=\delta_{s}+\alpha_{s} \delta_{r}$, together with $x^{*}$ satisfies the system (9), provided that we consider the constraints in $M$ except the one corresponding to $r$. Note that

$$
\phi-\sum_{s \in M-\{r\}} \widehat{\delta}_{s}=\phi-\sum_{s \in M} \delta_{s} .
$$

This process may be repeated until the system $M$ is reduced to a linearly independent subsystem. Thus, (9), with constraints only in this subsystem, are satisfied by the solution -in $x^{*}$ and multipliers - to (2). Since that subsystem has a unique solution, and the solution to (2) with this set of constraints exists, we conclude that this unique solution is $x^{*}, \phi, \widehat{\delta}$, and the lemma follows.

Now suppose that $A_{N}$ is spanned by the rest of the columns of $A$. As before, system (10) can be written as

$$
\begin{aligned}
\phi\left(\sum_{s \in M} a_{i s} \alpha_{s}\right)-\sum_{s \in M} a_{i s} \delta_{s} & =\frac{\mathcal{N}}{x_{i}^{*}} \Longleftrightarrow \\
\sum_{s \in M} a_{i s}\left(-\delta_{s}+\alpha_{s} \phi\right) & =\frac{\mathcal{N}}{x_{i}^{*}},
\end{aligned}
$$

for some vector $\alpha \in \mathbb{R}^{|M|}$, so that $\widehat{\phi}=0$ and $\widehat{\delta}_{s}=\delta_{s}-\alpha_{s} \phi$, together with $x^{*}$, is a new solution to system (9), when we consider only the constraints in $M$ except the one corresponding to $A$. Note that, again,

$$
-\sum_{s \in M} \widehat{\delta}_{s}=\phi-\sum_{s \in M} \delta_{s} .
$$

Repeating the procedure until we obtain a set of linearly independent constraints, and recalling that still a solution to (2) must exist and be characterized as the unique solution to (9) when considering the surviving constraints, we obtain that again that $\phi-\sum_{s \in M} \delta_{s}$ is unique. Then, Property P1 contradicts (11), since $\widehat{\delta}_{s} \geq 0$ for all $s \in M$. This finishes the proof of Step 2. 
Step 3 The solution $(\phi, \delta, x)$ to (2) with only equality constraints in (a linearly independent set of constraints) $M^{\prime}$ is a differentiable function of $v_{N}$ and $v_{s}$. Also, $d \delta_{s} / d v_{N}<0$ and $d \phi / d v_{N}>0$ for all $s \neq N$.

Proof. Consider the problem in (2) with only equality constraints in (a linearly independent set of constraints) $M^{\prime}$. Let $(\phi, \delta, x)$ be a solution to this problem. That is, $(\phi, \delta, x)$ maximizes

$$
L(\phi, \delta, x)=\Pi_{i} x_{i}-\phi\left(\sum_{i} x_{i}-v_{N}\right)+\sum_{s \in M^{\prime}} \delta_{s}\left(\sum_{i \in s} x_{i}-v_{s}\right) .
$$

The first order conditions for this problem are

$$
\begin{aligned}
G_{i} & \equiv \frac{\partial \mathcal{N}}{\partial x_{i}}-\phi+\sum_{s \ni i} \delta_{s}=0, \\
G_{s} & \equiv \sum_{i \in s} x_{i}-v_{s}=0, \\
G_{N} & \equiv-\sum_{i} x_{i}+v_{N}=0,
\end{aligned}
$$

where, once more, $\mathcal{N} \equiv \prod_{i \in e} x_{i}$. Differentiating this system with respect to $(\phi, \delta, x)$ and $v_{e}$, we obtain the system

$$
D \cdot\left[\begin{array}{l}
d x \\
d \delta \\
d \phi
\end{array}\right]=\left[-\frac{\partial G}{\partial v_{N}} d v_{N}\right],
$$

where $D$ is the Hessian matrix of $\mathcal{N}, H$, bordered with the Jacobian of the constraints, $A$ :

$$
D=\left[\begin{array}{cc}
H & A \\
A^{\prime} & 0
\end{array}\right]
$$

$d x$ is the vector of size $|N|$ with components $d x_{i} ; d \delta$ is the vector of dimension $m^{\prime}$ with components $d \delta_{s}$, and $\frac{\partial G}{\partial v_{N}}$ is the $|N|+m^{\prime}+1$ dimensional vector with terms $\frac{\partial G_{i}}{\partial v_{N}}, \frac{\partial G_{s}}{\partial v_{N}}$, and $\frac{\partial G_{N}}{\partial v_{N}}$. Note that $H$ is invertible. Indeed, the $i j$ entry in $H$ is $\frac{\mathcal{N}}{x_{i} x_{j}}$ if $i \neq j$ and 0 if $i=j$. Thus, multiplying each row $i$ by $\frac{x_{i}}{\mathcal{N}}$ and each column $j$ by $x_{i}$, all nonzero values, we have a matrix with entries 1 for all $i j$ with $i \neq j$ and 0 if $i=j$. The determinant of this matrix is $(-1)^{|N|-1}(|N|-1) \neq 0$, and so the determinant of $H$ is also nonzero. Thus,

$$
\operatorname{det} D=\operatorname{det} D / H \operatorname{det} H \text {, }
$$

where $D / H$ is the Schur complement of $H,-A^{\prime} H^{-1} A$. Note that $D / H$ is full rank. Indeed, $-H^{-1}$ is positive semidefinite and invertible, and so there exists a permutation matrix $P$ such that

$$
-P^{\prime} H^{-1} P=R^{\prime} R
$$


for some upper triangular matrix $R$ with all elements on the diagonal strictly positive (see, for instance, Highham 1990). Thus,

$$
-A^{\prime} H^{-1} A=-A^{\prime} P P^{\prime} H^{-1} P P^{\prime} A=-A^{\prime} P R^{\prime} R P^{\prime} A .
$$

Note that $A^{\prime} P$ is simply a permutation of $A^{\prime}$, and so has rank $m$, and then $A^{\prime} P R^{\prime}$ has also rank $m$. Thus, the rank of the product of that matrix and its transpose is $m$, and we conclude that $\operatorname{det} D / H>0$ and $\operatorname{det} D \neq 0$. Thus, $(\phi, \delta, x)$ is indeed a differentiable function of $v_{N}$ and of $v_{s}$.

Now, note that $\frac{\partial \mathcal{N}}{\partial x_{i}}=\frac{\mathcal{N}}{x_{i}}$. Thus, multiplying each of the expressions $G_{i}$ in the first order conditions of (12) by the corresponding $x_{i}$, and adding them for all $i$, and taking into account the rest of the first order conditions, we have

$$
n \mathcal{N}-\left(\phi v_{N}-\sum_{s \in M^{\prime}} \delta_{s}\right)=0 .
$$

This is an identity, and so, differentiating with respect to $\phi$ and $v_{N}$, and taking into account that (from the envelope theorem) $d \mathcal{N} / d v_{N}=\phi$ and $d \mathcal{N} / d \phi=0$, we obtain

$$
\frac{d \phi}{d v_{N}}=\frac{(n-1) \phi}{v_{N}}>0
$$

The rest of signs are obtained similarly.

For any game, we consider now a modification that makes Property P1 hold with equality:

Definition 9 For any game $(N, v)$, the game $(N, \widehat{v}(v))$ satisfies $\widehat{v}_{s}(v)=v_{s}$ for all $s \neq N$, and $\widehat{v}_{N}(v)$ is such that $\phi-\sum_{s \neq N} \delta_{s}=0$ in problem (2).

Lemma 10 For every game $(N, v)$ that either has a core with an empty interior or a non-empty interior but violates Property P1, $\widehat{v}(v)$ exists and is unique.

Proof. Consider any given game $v$ with a non-empty core such that Property 1 does not hold: $\phi-\sum_{s \neq e} \delta_{s}<0$. Consider the game $v^{\prime}$ defined by $v_{s}^{\prime}=v_{s}$ for all $s \neq N$, and $v_{N}^{\prime}=n \times v_{N}+\epsilon$. The solution to (9) for this game is $u_{i}=x_{i}=\frac{v_{N}^{\prime}}{n} \forall i$ (the generalized Nash bargaining solution), $\phi>0$, and $\delta_{s}=0$ for all $s \neq N$. Thus, $\phi-\sum_{s \neq N} \delta_{s}>0$. Since - some values of - the multipliers are continuous (and differentiable) in $v_{N}$ from Lemma 8 , and $\phi-\sum_{s \neq N} \delta_{s}$ is strictly monotone in $v_{N}$, the result follows. Now suppose the core of $v$ is empty. Still, the game $v_{N}^{\prime}$ has a non-empty core. Thus, there exists a minimum 
value $v_{N}^{\prime \prime}<v_{N}^{\prime}$ for which the core is not empty: the core is monotone in $v_{N}$. Thus, the result follows unless the game $v^{\prime \prime}$, defined with that value $v_{N}^{\prime \prime}$, satisfies Property $\mathrm{P} 1$ with strict inequality. We can rule out this possibility: the Nash product cannot increase if we increase the worth of a binding coalition by as much as what we increase the worth of $v_{N}$, when the interior of the core is empty.

Next, we provide necessary conditions for a SCOOP to be deterministic and nondeterministic, in terms on whether the game lies in Region I or in Region II.

Lemma 11 The SCOOP of a game that belongs to Region II cannot be deterministic. Moreover, if the game belongs to Region I, then the payoffs $\left\{u_{i}^{r}\right\}$ of a deterministic SCOOP, $\sigma=\left\{\left\{u_{i}^{s}\right\}_{i \in s},\left\{t_{i}^{s}\right\}_{i \in s}, p_{s},\left\{\lambda_{r}^{s}\right\}_{r \neq s}\right\}_{s \in S}$ is the solution to problem (2), and as a result $\sigma$ is the unique deterministic SCOOP in payoffs and probabilities.

Proof. Let $\sigma=\left\{\left\{u_{i}^{s}\right\}_{i \in s},\left\{t_{i}^{s}\right\}_{i \in s}, p_{s},\left\{\lambda_{r}^{s}\right\}_{r \neq s}\right\}_{s \in S}$ be a deterministic SCOOP with $p_{N}=$ 1. Then, it satisfies conditions (b) to (d) in Lemma 7. These conditions imply that $\left\{u_{i}^{s}\right\}_{i \in N}$ is an imputation in the core. Suppose the core of the game has a non-empty interior. Let $\mathcal{N}=\Pi_{i} u_{i}, x_{i}=u_{i}^{N}, \phi=\frac{\mathcal{N}}{\widetilde{\omega}}$, and $\delta_{s}=\lambda_{s}^{N} \frac{\mathcal{N}}{\widetilde{\omega}}$ for $s$ in (a subset of) $S_{0}(\sigma)$ (that forms a linearly independent system of constraints) and $\delta_{s}=0$ otherwise. These values satisfy condition (9) and hence the SCOOP coincides with the solution to problem (2), which is unique in $x$. Thus, since $\sum_{s \in S_{0}} \lambda_{s}^{N} \leq 1$ and $\phi-\sum_{s \neq N} \delta_{s}=\frac{N}{\widetilde{\omega}}\left(1-\sum_{s \neq N} \lambda_{s}^{N}\right) \geq 0$, Property P1 holds, and hence the game does not belong to Region II. Also, if the game belongs to Region I it is the unique deterministic SCOOP.

Lemma 12 The SCOOP of a game that belongs to Region I cannot be probabilistic. Moreover, if the game lies in Region II, then the payoffs $\left\{u_{i}\right\}$ of a probabilistic SCOOP, $\sigma=\left\{\left\{u_{i}^{s}\right\}_{i \in s},\left\{t_{i}^{s}\right\}_{i \in s}, p_{s},\left\{\lambda_{r}^{s}\right\}_{r \neq s}\right\}_{s \in S}$, with $\omega>0$, is the solution to problem (2) for the modified game $\widehat{v}(v)$, and hence $\sigma$ is unique in payoffs and probabilities.

Proof. Consider a game that lies in Region I, i.e., the solution to (9) satisfies $\phi-$ $\sum_{s \neq N} \delta_{s}>0$. Suppose that $\sigma=\left\{\left\{u_{i}^{s}\right\}_{i \in s},\left\{t_{i}^{s}\right\}_{i \in s}, p_{s},\left\{\lambda_{N}^{s}\right\}_{s \neq N}\right\}_{s \in S}$ is a probabilistic SCOOP for this game, and so satisfies conditions (a) to (d) of Lemma 6. Hence, we have that $\sum_{i} u_{i} \geq v_{N}$. If this weak inequality were to hold as equality, then we could construct a deterministic SCOOP with $p_{N}=1$ and $\lambda_{s}^{N}=p_{s}$, and using the arguments developed in the previous lemma, we would conclude that $\phi-\sum_{s \neq N} \delta_{s}=0$, which implies that the game does not lie in Region I. Therefore, $\sum_{i} u_{i}>v_{N}$. Now, consider a different game, $\widetilde{v}$, 
in which $\widetilde{v}_{N}=\sum_{i} u_{i}$ and $\widetilde{v}_{s}=v_{s}$, for all $s \neq N$. Note that the values $x_{i}=u_{i}$ for all $i$, $\phi=\frac{\mathcal{N}}{\omega}\left(1-p_{N}\right)$, and $\delta_{s}=p_{s} \frac{\mathcal{N}}{\omega}$, for $\mathcal{N}=\Pi_{i} u_{i}$, satisfy equations $(9)$, with $M=S_{+}(\sigma)$, for game $\widetilde{v}$. Thus, $\sum_{s \neq N} \delta_{s}=\sum_{s \neq N} p_{s} \frac{\mathcal{N}}{\omega}=\phi$. Note that if $v$ has a core with a nonempty interior, then $\widetilde{v}$ does too, and also a continuous path from $v$ to $\widetilde{v}$ with (weakly) increasing $v_{N}$. Thus, from Lemma 8 it must be the case that $\phi-\sum_{s \neq N} \delta_{s}<0$. This is a contradiction which proves the first part of the lemma.

Let us now prove generic uniqueness, i.e., uniqueness for games in Region II, so that if the game has a non-empty interior then $\phi-\sum_{s \neq N} \delta_{s}<0$. Suppose that we have two non-deterministic SCOOPs, $\sigma$ and $\sigma^{\prime}$. For a player $j$ who does not belong to any coalition in $S_{+}(\sigma)$, let $u_{j}=\omega$, and similarly for the SCOOP $\sigma^{\prime}$. Then, using the payoffs of $\sigma$, consider the game $\widetilde{v}$, where $\widetilde{v}_{N}=\sum_{i} u_{i}$ and $\widetilde{v}_{s}=v_{s}$ for all $s \neq N$; and, using the payoffs of $\sigma^{\prime}$, a similar game $\widetilde{v}^{\prime}$ with $\widetilde{v}_{N}^{\prime}=\sum_{i} u_{i}^{\prime}$. Note that the values $x_{i}=u_{i}, \phi=\frac{\mathcal{N}}{\omega}$, and $\delta_{s}=p_{s} \frac{\mathcal{N}}{\omega}$, where $\mathcal{N}=\Pi_{i} u_{i}$, satisfy (9) with $M=S_{+}(\sigma)$. Moreover, $\phi-\sum_{s \in M} \delta_{s}=0$, and so $\widetilde{v}=\widehat{v}(v)$. The same argument can be made for the SCOOP $\sigma^{\prime}$; in particular, $\widetilde{v}=\widehat{v}(v)$. Since $\widehat{v}(v)$ is unique according to Lemma 10, and the CNBS of a game is also unique, we conclude that $\left\{u_{i}\right\}=\left\{u_{i}^{\prime}\right\}$. Thus, the SCOOP is unique in payoffs. Also, note that an implication is that the SCOOP of a game that lies in Region II always assigns zero probability to the grand coalition, as $\sum_{i \in N} u_{i}=\widetilde{v}_{N}>v_{N}$. Also, as discussed in Lemma 6, the set of coalitions $M$ and the associated Lagrange multipliers are generically unique ${ }^{20}$, which implies that generically $p_{s}=p_{s}^{\prime}$. Thus, the SCOOP is also generically unique in probabilities. Even in those non-generic cases where the Lagrange multipliers are not unique, we still have that the individual probabilities of success are unique. In particular, condition (3) can be written as

$$
\frac{\mathcal{N}}{u_{i}}=\phi\left(1-\sum_{s \ni i} \frac{\delta_{s}}{\phi}\right)=\phi\left(1-q_{i}\right)
$$

for all $i$, and since $\frac{\mathcal{N}}{u_{i}}$ but also $\phi$ are unique from Lemma 8 , we conclude that $q_{i}=q_{i}^{\prime}$.

Once more, the arguments used in the proof also imply that conditions (a)-(d) in Lemma 6 are sufficient to (generically) identify the payoffs and probabilities of the unique SCOOP. Moreover, these conditions cannot be satisfied in Region I.

By putting together Lemmas 11 and 12, and then taking Theorem 1 into account, we conclude that the SCOOP is generically unique. Moreover: (a) In Region I the unique SCOOP (in payoffs and individual probabilities of success) is deterministic, and (b) in

\footnotetext{
${ }^{20}$ The cases where the Lagrange multipliers are not unique with $M$ constraints lie on a set of dimension $M-1$.
} 
Region II the unique SCOOP (in payoffs and individual probabilities of success) is nondeterministic.

\subsection{Non-cooperative implementation: Proof of Theorem 3}

We will limit attention to equilibria where players that are indifferent between accepting and rejecting in (2.2) (when the proposer strictly prefers acceptance) accept: if that were not so, then the proposer's best offer in 2.1 would not be defined, and that open set problem would be incompatible with equilibrium.

Suppose that we have a limit point of a sequence (in $\delta$ ) of equilibrium outcomes (payoffs and probabilities of coalitions' success). Let $x_{i}^{s}(\delta)$ be the expected payoff of player $i$ if coalition $s$ is selected in (1.2) and everybody accepts in (1.3), and $U_{i}(\delta)$ be the equilibrium payoff; that is, the expected payoff of player $i$ at 1 ) before the voting. We let $x_{i}^{s}$ and $U_{i}$ respectively to be the limits of these sequences. When there is no risk of ambiguity, we will drop the index $\delta$ from the corresponding variable.

Let $Z_{+}(\delta)$ be the set of coalitions $s$ that succeed with positive probability when the discount is $\delta$. Finally, let $Z_{+}$be the set of coalitions that succeed with positive probability in the limit. ${ }^{21}$ For $s \in Z_{+}(\delta)$, it must be that

$$
\begin{aligned}
x_{i}^{s}(\delta) & =\frac{1}{n_{s}}\left(v_{s}-\delta \sum_{j \neq i, j \in s} U_{j}(\delta)+\delta\left(n_{s}-1\right) U_{i}(\delta)\right) \\
& =\delta U_{i}(\delta)+\frac{1}{n_{s}}\left(v_{s}-\delta \sum_{, j \in s} U_{j}(\delta)\right),
\end{aligned}
$$

and so $x_{i}^{s}-\delta U_{i}(\delta)=x_{j}^{s}-\delta U_{j}(\delta)$ for all $i, j \in s$. Define $\Omega_{s}(\delta)=\frac{1}{n_{s}}\left(v_{s}-\delta \sum_{, j \in s} U_{j}(\delta)\right) \geq$ 0 , and accordingly $\Omega_{s}$ as the limit of $\Omega_{s}(\delta)$.

First, suppose that $Z_{+}$contains two disjoint coalitions, $s_{1}, s_{2} \in Z_{+}$with $s_{1} \cap s_{2}=\varnothing$. Consider a large $\delta$ (so that $s_{1}$ and $s_{2}$ are proposed with positive probability). Also, without loss of generality, assume that $\Omega_{s_{1}}(\delta) \leq \Omega_{s_{2}}(\delta)$. Consider $z=s_{1} \cup s_{2}$, and note that $n_{z}=n_{s_{1}}+n_{s_{2}}$. Also, note that, since the game is supperadditive, and so $v_{z} \geq v_{s_{1}}+v_{s_{2}}$ and so, from (14), $v_{z} \geq v_{s_{1}} \geq \sum_{i \in z} U_{i}(\delta)$, so that in (2.2) coalition $z$ would not be rejected; and also $n_{z} \Omega_{z}(\delta) \geq n_{s_{1}} \Omega_{s_{1}}(\delta)+n_{s_{2}} \Omega_{s_{2}}(\delta) \geq 0$. Thus,

$$
\begin{aligned}
\Omega_{s_{1}}(\delta)-\Omega_{z}(\delta) & \leq\left(1-\frac{n_{s_{1}}}{n_{z}}\right) \Omega_{s_{1}}(\delta)-\frac{n_{s_{2}}}{n_{z}} \Omega_{s_{2}}(\delta) \\
& =\frac{n_{s_{2}}}{n_{z}}\left(\Omega_{s_{1}}(\delta)-\Omega_{s_{2}}(\delta)\right) \leq 0
\end{aligned}
$$

\footnotetext{
${ }^{21}$ Note that we do not need to discount the probability of success.
} 
Thus, if in the limit $\Omega_{s_{1}}<\Omega_{s_{2}}$ then $\Omega_{s_{1}}-\Omega_{z}<0$. For sufficiently large $\delta$, and since for $s_{1} \in Z_{+}(\delta)$ we must have that $x_{i}^{s_{1}} \geq \delta x_{i}^{z}$, that is,

$$
\delta U_{i}(\delta)+\Omega_{s_{1}}(\delta) \geq \delta^{2} U_{i}(\delta)+\delta \Omega_{z}(\delta)
$$

we obtain a contradiction. (If $\Omega_{s_{1}}>\Omega_{s_{2}}$ we would have a similar contradiction, of course.)

A similar argument shows the other part: $\Omega_{s}$ cannot be larger for $s \notin Z_{+}$.

A consequence of this is that in equilibrium there could be no delays. Indeed, note that $\Omega_{s}(\delta)>0$ for all $s$ in $Z_{+}(\delta)$. Indeed, otherwise $x_{i}^{s}(\delta)=\delta U_{i}(\delta)$ for all such coalitions and players in them, which is a contradiction: the expected payoff $\left(U_{i}(\delta)\right)$ cannot be larger than the largest payoff received $\left(x_{i}^{s}(\delta)\right)$. Now, if $\Omega_{s}(\delta)>0$, then $v_{s}-\delta \sum_{, j \in s} U_{j}(\delta)>0$ for all coalitions in $Z_{+}(\delta)$, and so in equilibrium no player can reject in (1.3) or in (2.2).

Thus, let $\varphi_{s}(\delta)$ be the probability that coalition $s$ forms in the equilibrium when the discount is $\delta$, and let $\varphi_{s}$ be the limit point of that sequence. Define

$$
p_{s}=\varphi_{s}
$$

For the rest of the values that define a SCOOP, we consider two cases.

Case 1: Suppose that $\varphi_{s}<1$ for all $s \in S$. For all $s \in S$ define

$$
\lambda_{r}^{s}=\frac{\varphi_{r}}{1-\varphi_{s}}
$$

$u_{i}^{s}=x_{i}^{s}$ for all $s \in Z_{+}$(i.e., all $s$ with $p_{s}=\varphi_{s}>0$ ); $t_{i}^{s}$ according to (ii) in the definition of a SCOOP:

$$
t_{i}^{s}=\sum_{r \ni i, r \neq s} \lambda_{r}^{s} u_{i}^{r}
$$

Thus,

$$
t_{i}^{s}=\frac{U_{i}-\varphi_{s} x_{i}^{s}}{1-\varphi_{s}}
$$

which substituting in (14) in the limit implies for all $s \in Z_{+}$

$$
x_{i}^{s}=t_{i}^{s}+\frac{1}{n_{s}}\left(v_{s}-\sum_{j \in s} t_{j}^{s}\right) .
$$

For any $s \notin Z_{+}$, and given the above values (the only ones needed), define $u_{i}^{s}$ as in (i) of the definition of a SCOOP. Thus, for any such coalition, (with $p_{s}=0$, and so $t_{i}^{s}=U_{i}$ from (17)) $u_{i}^{s}$ is either 0 or $U_{i}+\Omega_{s}$. Therefore, the values thus define satisfy both parts (i) and (ii) of the definition of a SCOOP. Finally, since only coalitions with maximum $\Omega_{s}$ have positive probability of success, part (iii) of the definition of a SCOOP is also satisfied. 
Case 2: Finally, suppose that $\varphi_{s}=1$ for some $s \in S$. Consider any sequence of $\delta \rightarrow 1$ and define $\lambda_{r}^{s}$ as a limit point of $\frac{\delta \varphi_{r}(\delta)}{1-\delta \varphi_{s}(\delta)}$ for all $r \neq s$. (The sequence is well defined, as the denominator is positive for all terms of the sequence. Also, all these terms are smaller than 1 -and the sum over all $r \neq s$ is not larger than 1 . Thus, we have constructed a sequence in a compact set, so that indeed a limit point exists.) Also, define $\lambda_{s}^{r}=1$ for all $r, u_{i}^{s}=x_{i}^{s}$, and $t_{r}^{i}$ accordingly for all $r \neq s$. Then, define $u_{i}^{r}$ according to (i) in the definition of a SCOOP, and $t_{i}^{s}$ accordingly. The only thing left to prove is that $u_{i}^{s}$ satisfy (i) in the definition of a SCOOP, and that $u_{i}^{r} \leq u_{i}^{s}$.

To that end, note that $x_{i}^{s}\left(1-\sum_{r \in i, r \neq s} \lambda_{r}^{s}\right)$ is the same for all players in $s$. Indeed, recall that, from $(14), x_{i}^{s}-\delta U_{i}(\delta)=x_{j}^{s}-\delta U_{j}(\delta)$ for all $i, j \in s$. Also,

$$
\begin{aligned}
x_{i}^{s}(\delta)-\delta U_{i}(\delta) & =x_{i}^{s}(\delta)-\delta \sum_{r \ni i} \varphi_{r}(\delta) x_{i}^{r}(\delta) \\
& =x_{i}^{s}(\delta)\left(1-\delta \varphi_{s}(\delta)\right)-\delta \sum_{r \ni i, r \neq s} \varphi_{r}(\delta) x_{i}^{r}(\delta)
\end{aligned}
$$

Therefore,

$$
x_{i}^{s}(\delta)-\delta \sum_{r \ni i, r \neq s} \frac{\varphi_{r}(\delta)}{1-\delta \varphi_{s}(\delta)} x_{i}^{r}(\delta)
$$

is the same for all $i$ in $s$. Thus, at a limit point of the sequence $\frac{\varphi_{r}(\delta)}{1-\delta \varphi_{s}(\delta)}$, we have $x_{i}^{s}-$ $\sum_{r \ni i, r \neq s} \lambda_{r}^{s} x_{i}^{r}$, and the result follows if $x_{i}^{s}=x_{i}^{r}$ when $\lambda_{r}^{s}>0$. This, in turn, holds since, otherwise, i.e., if $\left|x_{i}^{s}-x_{i}^{r}\right|>\epsilon$, for large $\delta$ (when $\varphi_{r}(\delta)>0$ ) player $i$ would have an incentive to reject in (1.3) either $s$ or $r$ and propose the other in the following period. Thus, $x_{i}^{s}\left(1-\sum_{r \in i, r \neq s} \lambda_{r}^{s}\right)$ is the same for al players in $s$, and since $\sum_{i \in s} x_{i}^{s}=v_{s}$, it follows that $u_{i}^{s}$ satisfy (i) in the definition of a SCOOP. Finally, $u_{i}^{r} \leq u_{i}^{s}$ follows exactly from the same argument as in case 1).

This concludes the proof of the first part of the theorem: any converging selection of SPE in stationary strategies implements the SCOOP asymptotically.

We now show that a sequence of equilibria - with positive probability of a coalition forming- exists. Begin with the SCOOP values for $u_{i}$ and $\omega$ and let $Z_{+}=S_{+}$. Note that (14) implies a modified version of the equal loss property. Indeed, for any two players in $s$, the last term is common. We begin by investigating the existence of a solution $\left\{\varphi_{s}\right\}_{s \in S_{+}}, \Omega$ to the system.

$$
\begin{aligned}
u_{i}\left(1-\delta \sum_{s \ni i, s \in S_{+}} \varphi_{s}\right) & =\Omega, \quad \forall i \in \cup S_{+} \\
\sum_{s \in S_{+}} \varphi_{s} & =1,
\end{aligned}
$$


for given $\delta$. That is, we prove that the following linear system has a positive solution

$$
C_{+} \cdot\left[\begin{array}{l}
\Omega \\
\varphi
\end{array}\right]=\left[\begin{array}{l}
u \\
1
\end{array}\right],
$$

where $u$ is the $N$-dimensional vector with components $u_{i}$, and $\varphi$ is the $m$-dimensional vector with components $\varphi_{s}$, with $m=\left|S_{+}\right|$, and $C_{+}$is the $(N+1) \times(m+1)$ matrix

$$
C_{+}=\left[\begin{array}{cccc}
1 & \delta I\left(1, s_{1}\right) u_{1} & \ldots & \delta I\left(1, s_{m}\right) u_{1} \\
\ldots & \ldots & \ldots & \ldots \\
1 & \delta I\left(N, s_{1}\right) u_{N} & \ldots & \delta I\left(N, s_{m}\right) u_{N} \\
0 & 1 & . . & 1
\end{array}\right]
$$

where $I(i, s)$ is an indicator function that takes the value of 1 if $i \in s$ and 0 otherwise. Note that, for $\delta=1$, this system has a solution: the probabilities $p_{s}$ and the "loss" $\Omega$ in the SCOOP. Denote this solution by $\left(\Omega^{*}, \varphi^{*}\right)$. Also note that the space spanned by the rows of the matrix $C_{+}$is the same as the one spanned by those of the matrix $\widehat{C}_{+}$obtained by multiplying each of its $N$ rows by $1 / \delta$. $(u, 1)$ belongs to this space: in this basis $\widehat{C}_{+}$, $(u, 1)$ has coordinates $\left(\delta \Omega^{*}, \varphi^{*}\right)$. Thus, $(u, 1)$ belongs to the space spanned by $C_{+}$and so a solution to the system (19) indeed exists. Moreover, since $\varphi_{s}^{*}>0$, the new coordinates $(\Omega, \varphi)$ are all positive for sufficiently large $\delta$.

Thus, for large, given $\delta$, consider the following strategy for player $i$ for a given value of $\delta$, defined in terms of the $\operatorname{SCOOP}$ values $u$ and this solution $(\Omega, \varphi)$, defining $U_{j}=$ $\sum_{s \ni j} \varphi_{s} u_{j}$ :

- $b_{s}^{i}=\varphi_{s}$ if $i \in s \in S_{+} ; b_{s}^{i}=0$ if $i \in s \notin S_{+}$.

- Accept in (1.3) if $v_{s} \geq \delta \sum_{j \in s} u_{j}$; reject otherwise. (Reject otherwise and choose any coalition in $S_{+}$containing $i$ in the next period.)

- In (2.1), if selected, propose keeping $v_{s}-\delta \sum_{\substack{, j \in s \\ j \neq i}} U_{j}$, and giving $U_{j}$ to each $j \neq i$, if $v_{s} \geq \delta \sum_{, j \in s} U_{j}$ and otherwise, insist on keeping $v_{s}$.

- In (2.2), accept if proposed to receive $\delta U_{i}$ or more, and reject otherwise.

Given that all players play according to this strategy, the behavior stipulated for (2) is optimal. So the strategy profile is a Nash equilibrium in any subgame beginning at any node of (2). Also, if a player rejects in (1.3), choosing any $s \in S_{+}$with $i \in s$ is also optimal: the player will get the highest feasible payoff $u_{i}$. That is also what the player will obtain accepting in (1.3) any coalition where $v_{s} \geq \delta \sum_{j \in s} u_{j}$. If $v_{s}<\delta \sum_{j \in s} u_{j}$, the 
optimal response will be to reject and (hope to) choose in the next period a coalition in $S_{+}$. Finally, the ballot $b_{s}^{i}$ is also optimal. Indeed, a (one-shot) deviation cannot result in a higher payoff: either it does not affect the probability that a coalition in $S_{+}$containing $i$ is selected by Nature, or it reduces it. Any coalition not in $S_{+}$has probability 0 independent of what the player votes. Thus, for large $\delta$ indeed a SPE in stationary strategies indeed exists.

For simplicity, in the existence part of the proof of this result we have used equilibrium strategies where players may not exhaust all their votes in the voting stage. In particular, $\sum_{s \ni i} b_{i}^{s}$ is typically strictly less than one. It is important to note that these voting strategies may be part of weakly non dominated strategies of the game. Nevertheless, it can be shown (details upon request) that there exists equilibrium strategies that exhaust the players' voting ability and support the same probabilities and payoffs. 